\title{
About Graph Mappings
}

\author{
Sebastian Koch \\ Johannes Gutenberg University \\ Mainz, Germany ${ }^{1}$
}

\begin{abstract}
Summary. In this articles adjacency-preserving mappings from a graph to another are formalized in the Mizar system [7, 2]. The generality of the approach seems to be largely unpreceeded in the literature to the best of the author's knowledge. However, the most important property defined in the article is that of two graphs being isomorphic, which has been extensively studied. Another graph decorator is introduced as well.
\end{abstract}

MSC: 05C60 68T99 03B35

Keywords: graph homomorphism; graph isomorphism

MML identifier: GLIB_010, version: 8.1.09 5.59.1363

\section{INTRODUCTION}

Writing this article has been rather challenging. "Much of graph theory is concerned with the study of simple graphs" [3, p. 3], so most graph theory books are only concerned with graph homomorphisms between simple graphs, if they are concerned with anything more general than isomorphisms at all. [3] writes about general graphs; isomorphisms are done in the first chapter while homomorphisms are only looked at in the context of vertex colorings in chapter 14. The book "Graphs and homomorphisms" [8] only handles (di)graphs without multiple parallel edges. The book "Graph coloring problems" [10] notes homomorphisms between loopless graphs, but doesn't elaborate. [6] only handles homomorphisms between simple graphs. [14] shortly describes homomorphisms between undirected graphs. 9] handles homomorphisms between

\footnotetext{
${ }^{1}$ The author is enrolled in the Johannes Gutenberg University in Mayence, Germany, mailto: skoch02@students . uni-mainz.de 
digraphs without parallel edges. [16] writes about general graphs but, like most graph books, only about isomorphisms. The best source so far has been [11], where graph homomorphisms are introduced for digraphs possibly containing loops and multiple parallel edges (just like graphs are formalized in [15]) but the focus is almost immediately shifted to homomorphisms between simple graphs. So a quick overview of the formalized notation seems in order.

A graph $G$ consists of a non empty set $V(G)$ called vertices of $G$, a set $E(G)$ called edges of $G$ and two functions $s(G), t(G): E(G) \rightarrow V(G)$, the source and target of $G$. For $e \in E(G), v, w \in V(G)$ we write $e$ joins $v$ to $w$ if $s(G)(e)=v$ and $t(G)(e)=w$, and we write $e$ joins $v$ and $w$ if $e$ joins $v$ to $w$ or $e$ joins $w$ to $v$. Let $G_{1}, G_{2}$ be graphs. A partial graph mapping from $G_{1}$ to $G_{2}$ is an ordered pair $F=\left\langle F_{\mathbb{V}}, F_{\mathbb{E}}\right\rangle$ with the following properties:

- $F_{\mathbb{V}}$ is a partial function from $V\left(G_{1}\right)$ to $V\left(G_{2}\right)$.

- $F_{\mathbb{E}}$ is a partial function from $E\left(G_{1}\right)$ to $E\left(G_{2}\right)$.

- For any $e \in \operatorname{dom} F_{\mathbb{E}}$ holds $s(G)(e), t(G)(e) \in \operatorname{dom} F_{\mathbb{V}}$.

- For any $e \in \operatorname{dom} F_{\mathbb{E}}$ and $v, w \in \operatorname{dom} F_{\mathbb{V}}$ such that $e$ joins $v$ and $w$ holds $F_{\mathbb{E}}(e)$ joins $F_{\mathbb{V}}(v)$ and $F_{\mathbb{V}}(w)$.

Note that $\langle f, \emptyset\rangle$ is a valid partial graph mapping for any partial function $f$ : $V\left(G_{1}\right) \rightarrow V\left(G_{2}\right)$, especially for $f=\emptyset$. Now define the following attributes:

- $F$ is empty if dom $F_{\mathbb{V}}=\emptyset$.

- $F$ is total (or a homomorphism) if $\operatorname{dom} F_{\mathbb{V}}=V\left(G_{1}\right)$ and $\operatorname{dom} F_{\mathbb{E}}=E\left(G_{1}\right)$.

- $F$ is onto (or surjective) if $\operatorname{rng} F_{\mathbb{V}}=V\left(G_{2}\right)$ and $\operatorname{rng} F_{\mathbb{E}}=E\left(G_{2}\right)$.

- $F$ is one-to-one (or injective) if $F_{\mathbb{V}}$ and $F_{\mathbb{E}}$ are.

- $F$ is semi-continuous if for any $e \in \operatorname{dom} F_{\mathbb{E}}$ and $v, w \in \operatorname{dom} F_{\mathbb{V}}$ such that $F_{\mathbb{E}}(e)$ joins $F_{\mathbb{V}}(v)$ and $F_{\mathbb{V}}(w)$ holds $e$ joins $v$ and $w$.

- $F$ is continuous if for any $\tilde{e} \in E\left(G_{2}\right)$ and $v, w \in \operatorname{dom} F_{\mathbb{V}}$ such that $\tilde{e}$ joins $F_{\mathbb{V}}(v)$ and $F_{\mathbb{V}}(w)$ exists an $e \in \operatorname{dom} F_{\mathbb{E}}$ such that $F_{\mathbb{E}}(e)=\tilde{e}$ and $e$ joins $v$ and $w$.

- $F$ is a weak subgraph-embedding if it is total and one-to-one.

- $F$ is a strong subgraph-embedding if it is total, one-to-one and continuous.

- $F$ is an isomorphism if it is total, one-to-one and onto.

Because modes in Mizar must always be inhabitated, partial graph mappings are the chosen foundation rather than homomorphisms, which may not exist between two graphs. The attributes total, onto and one-to-one were named like their function analogons from [4] and [5]. The continuous attribute was inspired by the continuous vertex mappings of [11] and is in fact sometimes different from semi-continuous. Semi-continuous seemed like the natural generalization 
of continuous for graph mappings instead of vertex mappings, but that turned out to be false. Still, a semi-continuous graph mapping already carries a lot of properties from $G_{1}$ to $G_{2}$, so the definition was kept. Corresponding attributes for directed graph mappings are given in this article as well.

If $F$ is a weak subgraph-embedding, then $G_{1}$ is isomorphic to a subgraph of $G_{2}$. If $F$ is a strong subgraph-embedding, then $G_{1}$ is isomorphic to an induced subgraph of $G_{2}$. The short term embedding was desperately avoided to be available for embeddings of graphs into the plane and other surfaces. If $F$ is one-to-one, it is also semi-continuous. If $F$ is semi-continuous and onto, it is also continuous.

Originally, only an article about graph isomorphisms was planned, but it was changed to provide a solid foundation of general graph mappings. Now this article also includes the restriction of $F$ to subgraphs of $G_{1}$ or $G_{2}$, the domain and range of $F$ defined as the plain subgraphs of $G_{1}$ and $G_{2}$ induced by dom $F_{\mathbb{V}}$, $\operatorname{dom} F_{\mathbb{E}}$ and $\operatorname{rng} F_{\mathbb{V}}, \operatorname{rng} F_{\mathbb{E}}$ respectively, and the images of walks under $F$. Of course the inverse of $F$ and the composition of two graph mappings are included as well.

Additionally, the ordering of a graph, which is just an enumeration of its vertices, has been introduced as yet another graph decorator. This decorator is planned as a tool to identify graphs with trees from [1]. Attributes describing if $F$ preserves the weights, edge labels, vertex labels or the ordering have been added as well.

\section{Preliminaries}

Now we state the propositions:

(1) Let us consider functions $A, B, C, D$. Suppose $D \cdot A=C \uparrow \operatorname{dom} A$. Then $(D\lceil\operatorname{dom} B) \cdot A=C \uparrow \operatorname{dom}(B \cdot A)$.

Proof: Set $f=(D \uparrow \operatorname{dom} B) \cdot A$. Set $g=C \uparrow \operatorname{dom}(B \cdot A)$. For every object $x$ such that $x \in \operatorname{dom} f$ holds $f(x)=g(x)$.

(2) Let us consider a one-to-one function $A$, and functions $C, D$. Suppose $D \cdot A=C\left\lceil\operatorname{dom} A\right.$. Then $C \cdot\left(A^{-1}\right)=D\left\lceil\operatorname{dom}\left(A^{-1}\right)\right.$.

Proof: For every object $y, y \in \operatorname{dom}\left(C \cdot\left(A^{-1}\right)\right)$ iff $y \in \operatorname{dom}\left(D\left\lceil\operatorname{dom}\left(A^{-1}\right)\right)\right.$. For every object $y$ such that $y \in \operatorname{dom}\left(C \cdot\left(A^{-1}\right)\right)$ holds $\left(C \cdot\left(A^{-1}\right)\right)(y)=$ $\left(D\left\lceil\operatorname{dom}\left(A^{-1}\right)\right)(y)\right.$.

Let $G$ be a non finite graph and $X$ be a set. One can verify that

$G$.set(WeightSelector, $X$ ) is non finite and $G$.set(ELabelSelector, $X$ ) is non finite and $G$.set(VLabelSelector, $X$ ) is non finite.

Let $G$ be a non loopless graph. One can check that $G$.set(WeightSelector, $X$ ) is non loopless and $G$.set(ELabelSelector, $X$ ) is non loopless and 
$G$.set(VLabelSelector, $X$ ) is non loopless.

Let $G$ be a non non-multi graph. Note that $G$.set(WeightSelector, $X$ ) is non non-multi and $G$.set(ELabelSelector, $X$ ) is non non-multi and

$G$.set(VLabelSelector, $X$ ) is non non-multi. Let $G$ be a non non-directedmulti graph. Let us note that $G$.set(WeightSelector, $X$ ) is non non-directedmulti and $G$.set(ELabelSelector, $X$ ) is non non-directed-multi and

$G$.set(VLabelSelector, $X$ ) is non non-directed-multi.

Let $G$ be a non connected graph. Observe that $G$.set(WeightSelector, $X$ ) is non connected and $G$.set(ELabelSelector, $X$ ) is non connected and

$G$.set(VLabelSelector, $X$ ) is non connected.

Let $G$ be a non acyclic graph. Let us observe that $G$.set(WeightSelector, $X$ ) is non acyclic and $G$.set(ELabelSelector, $X$ ) is non acyclic and

$G$.set(VLabelSelector, $X$ ) is non acyclic. Let $G$ be a graph. We say that $G$ is elabel-full if and only if

(Def. 1) ELabelSelector $\in \operatorname{dom} G$ and there exists a many sorted set $f$ indexed by the edges of $G$ such that $G$ (ELabelSelector) $=f$.

We say that $G$ is vlabel-full if and only if

(Def. 2) VLabelSelector $\in \operatorname{dom} G$ and there exists a many sorted set $f$ indexed by the vertices of $G$ such that $G$ (VLabelSelector) $=f$.

Let us observe that every graph which is elabel-full is also elabeled and every graph which is vlabel-full is also vlabeled.

Let $G$ be an e-graph. We say that $G$ is elabel-distinct if and only if

(Def. 3) the elabel of $G$ is one-to-one.

Let $G$ be a v-graph. We say that $G$ is vlabel-distinct if and only if

(Def. 4) the vlabel of $G$ is one-to-one.

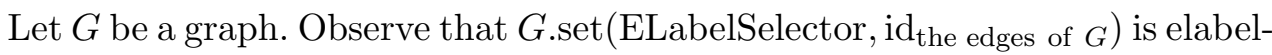

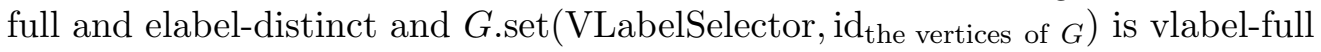
and vlabel-distinct and there exists an e-graph which is elabel-distinct and elabel-full and there exists a v-graph which is vlabel-distinct and vlabel-full.

Let $G$ be an elabel-full graph. Let us observe that the elabel of $G$ yields a many sorted set indexed by the edges of $G$. Let $G$ be a vlabel-full graph. Observe that the vlabel of $G$ yields a many sorted set indexed by the vertices of $G$. Let $G$ be an elabel-distinct e-graph. Let us note that the elabel of $G$ is one-to-one.

Let $G$ be a vlabel-distinct v-graph. Observe that the vlabel of $G$ is one-toone. Let $G$ be an elabel-full graph and $X$ be a set. One can verify that

$G$.set(WeightSelector, $X$ ) is elabel-full and $G$.set(VLabelSelector, $X$ ) is elabelfull. Let $G$ be a vlabel-full graph. One can check that $G$.set(WeightSelector, $X$ ) is vlabel-full and $G$.set(ELabelSelector, $X$ ) is vlabel-full. 
Let $G$ be an elabel-distinct e-graph. Note that $G$.set(WeightSelector, $X$ ) is elabel-distinct and $G$.set(VLabelSelector, $X$ ) is elabel-distinct.

Let $G$ be a vlabel-distinct v-graph. Let us observe that $G$.set(WeightSelector,

$X$ ) is vlabel-distinct and $G$.set(ELabelSelector, $X$ ) is vlabel-distinct and there exists an ev-graph which is elabel-full, elabel-distinct, vlabel-full, and vlabeldistinct.

Let $G_{1}$ be a w-graph, $E$ be a set, and $G_{2}$ be a graph given by reversing directions of the edges $E$ of $G_{1}$. Observe that $G_{2}$.set(WeightSelector, the weight of $G_{1}$ ) is weighted.

Let $G_{1}$ be an e-graph. One can verify that $G_{2}$.set(ELabelSelector, the elabel of $G_{1}$ ) is elabeled.

Let $G_{1}$ be a v-graph, $V$ be a set, and $G_{2}$ be a graph given by reversing directions of the edges $V$ of $G_{1}$. Observe that $G_{2}$.set(VLabelSelector, the vlabel of $G_{1}$ ) is vlabeled.

Let $G_{1}$ be an elabel-full graph, $E$ be a set, and $G_{2}$ be a graph given by reversing directions of the edges $E$ of $G_{1}$. Note that $G_{2}$.set(ELabelSelector, the elabel of $G_{1}$ ) is elabel-full.

Let $G_{1}$ be a vlabel-full graph, $V$ be a set, and $G_{2}$ be a graph given by reversing directions of the edges $V$ of $G_{1}$. Note that $G_{2}$.set(VLabelSelector, the vlabel of $G_{1}$ ) is vlabel-full. Let $G_{1}$ be an elabel-distinct e-graph, $E$ be a set, and $G_{2}$ be a graph given by reversing directions of the edges $E$ of $G_{1}$. Note that $G_{2}$.set(ELabelSelector, the elabel of $G_{1}$ ) is elabel-distinct. Let $G_{1}$ be a vlabeldistinct v-graph. Observe that $G_{2}$.set (VLabelSelector, the vlabel of $G_{1}$ ) is vlabeldistinct.

\section{Ordering of A Graph}

The functor OrderingSelector yielding an element of $\mathbb{N}$ is defined by the term (Def. 5) 8 .

Let $G$ be a graph structure. We say that $G$ is ordered if and only if

(Def. 6) OrderingSelector $\in \operatorname{dom} G$ and $G$ (OrderingSelector) is an enumeration of the vertices of $G$.

Let $G$ be a graph and $X$ be a set. Note that $G$.set(OrderingSelector, $X$ ) is graph-like and $G$.set(OrderingSelector, $X$ ) is non plain.

Let $G$ be a w-graph. One can verify that $G$.set(OrderingSelector, $X$ ) is weighted.

Let $G$ be an e-graph. One can check that $G$.set(OrderingSelector, $X$ ) is elabeled.

Let $G$ be a v-graph. Note that $G$.set(OrderingSelector, $X$ ) is vlabeled. 
Let $G$ be a graph and $X$ be an enumeration of the vertices of $G$. Note that $G$.set(OrderingSelector, $X$ ) is ordered and there exists a graph structure which is graph-like, weighted, elabeled, vlabeled, and ordered.

Let $G$ be an ordered graph. The ordering of $G$ yielding an enumeration of the vertices of $G$ is defined by the term

(Def. 7) $G$ (OrderingSelector).

Now we state the proposition:

(3) Let us consider a graph $G$, and a set $X$.

Then $G \approx G$.set(OrderingSelector, $X$ ).

Let $G$ be an elabel-full graph and $X$ be a set. Let us note that

$G$.set(OrderingSelector, $X$ ) is elabel-full.

Let $G$ be a vlabel-full graph. Let us note that $G$.set(OrderingSelector, $X$ ) is vlabel-full.

Let $G$ be an elabel-distinct e-graph. Let us note that $G$.set(OrderingSelector,

$X)$ is elabel-distinct.

Let $G$ be a vlabel-distinct v-graph. Observe that $G$.set(OrderingSelector, $X$ ) is vlabel-distinct.

Let $G$ be a finite graph. Let us observe that $G$.set(OrderingSelector, $X$ ) is finite.

Let $G$ be a non finite graph. Let us observe that $G$.set(OrderingSelector, $X$ ) is non finite.

Let $G$ be a loopless graph. Let us observe that $G$.set(OrderingSelector, $X$ ) is loopless.

Let $G$ be a non loopless graph. Let us observe that $G$.set(OrderingSelector, $X$ ) is non loopless.

Let $G$ be a trivial graph. Let us observe that $G$.set(OrderingSelector, $X$ ) is trivial.

Let $G$ be a non trivial graph. Let us observe that $G$.set(OrderingSelector, $X$ ) is non trivial.

Let $G$ be a non-multi graph. Let us observe that $G$.set(OrderingSelector, $X$ ) is non-multi.

Let $G$ be a non non-multi graph. Let us observe that

$G$.set(OrderingSelector, $X$ ) is non non-multi.

Let $G$ be a non-directed-multi graph. Let us observe that

$G$.set(OrderingSelector, $X$ ) is non-directed-multi.

Let $G$ be a non non-directed-multi graph. Let us observe that

$G$.set(OrderingSelector, $X$ ) is non non-directed-multi.

Let $G$ be a connected graph. Let us observe that $G$.set(OrderingSelector, $X$ ) is connected. 
Let $G$ be a non connected graph. Let us note that $G$.set(OrderingSelector, $X$ ) is non connected.

Let $G$ be an acyclic graph. Let us note that $G$.set(OrderingSelector, $X$ ) is acyclic.

Let $G$ be a non acyclic graph. One can check that $G$.set(OrderingSelector, $X$ ) is non acyclic.

Let $G$ be an edgeless graph. One can check that $G$.set(OrderingSelector, $X$ ) is edgeless.

Let $G$ be a non edgeless graph. Let us observe that $G$.set(OrderingSelector, $X$ ) is non edgeless.

Let $G$ be an ordered graph. Let us observe that $G$.set(WeightSelector, $X$ ) is ordered and $G$.set(ELabelSelector, $X$ ) is ordered and $G$.set(VLabelSelector, $X$ ) is ordered.

Let $G_{1}$ be an ordered graph and $G_{2}$ be a spanning subgraph of $G_{1}$. Note that $G_{2}$.set(OrderingSelector, the ordering of $G_{1}$ ) is ordered.

Let $E$ be a set and $G_{2}$ be a graph given by reversing directions of the edges $E$ of $G_{1}$. Let us observe that $G_{2}$.set(OrderingSelector, the ordering of $G_{1}$ ) is ordered.

\section{Graph Mappings}

Let $G_{1}, G_{2}$ be graphs. A partial graph mapping from $G_{1}$ to $G_{2}$ is an object defined by

(Def. 8) there exist functions $f, g$ such that $i t=\langle f, g\rangle$ and $\operatorname{dom} f \subseteq$ the vertices of $G_{1}$ and $\operatorname{rng} f \subseteq$ the vertices of $G_{2}$ and dom $g \subseteq$ the edges of $G_{1}$ and $\operatorname{rng} g \subseteq$ the edges of $G_{2}$ and for every object $e$ such that $e \in \operatorname{dom} g$ holds (the source of $\left.G_{1}\right)(e)$, (the target of $\left.G_{1}\right)(e) \in \operatorname{dom} f$ and for every objects $e, v, w$ such that $e \in \operatorname{dom} g$ and $v, w \in \operatorname{dom} f$ holds if $e$ joins $v$ and $w$ in $G_{1}$, then $g(e)$ joins $f(v)$ and $f(w)$ in $G_{2}$.

Let us observe that every partial graph mapping from $G_{1}$ to $G_{2}$ is pair.

Let $F$ be a partial graph mapping from $G_{1}$ to $G_{2}$. We introduce the notation $F_{\mathbb{V}}$ as a synonym of $(F)_{\mathbf{1}}$ and $F_{\mathbb{E}}$ as a synonym of $(F)_{\mathbf{2}}$.

One can check that $\left\langle F_{\mathbb{V}}, F_{\mathbb{E}}\right\rangle$ reduces to $F$.

One can verify that $F_{\mathbb{V}}$ is function-like and relation-like as a set and $F_{\mathbb{E}}$ is function-like and relation-like as a set and $F_{\mathbb{V}}$ is (the vertices of $G_{1}$ )-defined and (the vertices of $G_{2}$ )-valued as a function and $F_{\mathbb{E}}$ is (the edges of $G_{1}$ )-defined and (the edges of $G_{2}$ )-valued as a function.

Note that the functor $F_{\mathbb{V}}$ yields a partial function from the vertices of $G_{1}$ to the vertices of $G_{2}$. Observe that the functor $F_{\mathbb{E}}$ yields a partial function from the edges of $G_{1}$ to the edges of $G_{2}$. Now we state the proposition: 
(4) Let us consider graphs $G_{1}, G_{2}$, a partial graph mapping $F$ from $G_{1}$ to $G_{2}$, and objects $e, v, w$. Suppose $e \in \operatorname{dom}\left(F_{\mathbb{E}}\right)$ and $v, w \in \operatorname{dom}\left(F_{\mathbb{V}}\right)$. If $e$ joins $v$ and $w$ in $G_{1}$, then $\left(F_{\mathbb{E}}\right)(e)$ joins $\left(F_{\mathbb{V}}\right)(v)$ and $\left(F_{\mathbb{V}}\right)(w)$ in $G_{2}$.

Let us consider graphs $G_{1}, G_{2}$, a partial graph mapping $F$ from $G_{1}$ to $G_{2}$, and an object $e$. Now we state the propositions:

(5) Suppose $e \in \operatorname{dom}\left(F_{\mathbb{E}}\right)$. Then (the source of $\left.G_{1}\right)(e)$, (the target of $\left.G_{1}\right)(e) \in$ $\operatorname{dom}\left(F_{\mathbb{V}}\right)$.

(6) Suppose $e \in \operatorname{rng} F_{\mathbb{E}}$. Then (the source of $\left.G_{2}\right)(e)$, (the target of $\left.G_{2}\right)(e) \in$ $\operatorname{rng} F_{\mathbb{V}}$. The theorem is a consequence of (5) and (4).

(7) Let us consider graphs $G_{1}, G_{2}$, and a partial graph mapping $F$ from $G_{1}$ to $G_{2}$. Then

(i) $\operatorname{dom}\left(F_{\mathbb{E}}\right) \subseteq G_{1}$.edgesBetween $\left(\operatorname{dom}\left(F_{\mathbb{V}}\right)\right)$, and

(ii) $\operatorname{rng} F_{\mathbb{E}} \subseteq G_{2}$.edgesBetween $\left(\operatorname{rng} F_{\mathbb{V}}\right)$.

PROOF: For every object $e$ such that $e \in \operatorname{dom}\left(F_{\mathbb{E}}\right)$ holds

$e \in G_{1}$.edgesBetween $\left(\operatorname{dom}\left(F_{\mathbb{V}}\right)\right)$. For every object $e$ such that $e \in \operatorname{rng} F_{\mathbb{E}}$ holds $e \in G_{2}$.edgesBetween $\left(\operatorname{rng} F_{\mathbb{V}}\right)$.

(8) Let us consider graphs $G_{1}, G_{2}$, a partial function $f$ from the vertices of $G_{1}$ to the vertices of $G_{2}$, and a partial function $g$ from the edges of $G_{1}$ to the edges of $G_{2}$. Suppose for every object $e$ such that $e \in \operatorname{dom} g$ holds (the source of $\left.G_{1}\right)(e)$, (the target of $\left.G_{1}\right)(e) \in \operatorname{dom} f$ and for every objects $e, v, w$ such that $e \in \operatorname{dom} g$ and $v, w \in \operatorname{dom} f$ holds if $e$ joins $v$ and $w$ in $G_{1}$, then $g(e)$ joins $f(v)$ and $f(w)$ in $G_{2}$. Then $\langle f, g\rangle$ is a partial graph mapping from $G_{1}$ to $G_{2}$.

Let us consider graphs $G_{1}, G_{2}, G_{3}, G_{4}$ and a partial graph mapping $F$ from $G_{1}$ to $G_{2}$. Now we state the propositions:

(9) If $G_{1} \approx G_{3}$ and $G_{2} \approx G_{4}$, then $F$ is a partial graph mapping from $G_{3}$ to $G_{4}$. The theorem is a consequence of (5), (4), and (8).

(10) Suppose there exist sets $E_{1}, E_{2}$ such that $G_{3}$ is a graph given by reversing directions of the edges $E_{1}$ of $G_{1}$ and $G_{4}$ is a graph given by reversing directions of the edges $E_{2}$ of $G_{2}$. Then $F$ is a partial graph mapping from $G_{3}$ to $G_{4}$. The theorem is a consequence of (5), (4), and (8).

Let $G$ be a graph. The functor $\operatorname{id}_{G}$ yielding a partial graph mapping from $G$ to $G$ is defined by the term

(Def. 9) $\left\langle\operatorname{id}_{\alpha}, \operatorname{id}_{\beta}\right\rangle$, where $\alpha$ is the vertices of $G$ and $\beta$ is the edges of $G$.

Now we state the propositions:

(11) Let us consider graphs $G_{1}, G_{2}$. Suppose $G_{1} \approx G_{2}$. Then

(i) $\operatorname{id}_{G_{1}}=\mathrm{id}_{G_{2}}$, and 
(ii) $\operatorname{id}_{G_{1}}$ is a partial graph mapping from $G_{1}$ to $G_{2}$.

The theorem is a consequence of (9).

(12) Let us consider a graph $G_{1}$, a set $E$, and a graph $G_{2}$ given by reversing directions of the edges $E$ of $G_{1}$. Then

(i) $\operatorname{id}_{G_{1}}=\mathrm{id}_{G_{2}}$, and

(ii) $\operatorname{id}_{G_{1}}$ is a partial graph mapping from $G_{1}$ to $G_{2}$.

Proof: There exist sets $E_{1}, E_{2}$ such that $G_{1}$ is a graph given by reversing directions of the edges $E_{1}$ of $G_{1}$ and $G_{2}$ is a graph given by reversing directions of the edges $E_{2}$ of $G_{1}$.

Let $G_{1}, G_{2}$ be graphs and $F$ be a partial graph mapping from $G_{1}$ to $G_{2}$. We say that $F$ is empty if and only if

(Def. 10) $\operatorname{dom}\left(F_{\mathbb{V}}\right)$ is empty.

We say that $F$ is total if and only if

(Def. 11) $\operatorname{dom}\left(F_{\mathbb{V}}\right)=$ the vertices of $G_{1}$ and $\operatorname{dom}\left(F_{\mathbb{E}}\right)=$ the edges of $G_{1}$.

We say that $F$ is onto if and only if

(Def. 12) $\operatorname{rng} F_{\mathbb{V}}=$ the vertices of $G_{2}$ and $\operatorname{rng} F_{\mathbb{E}}=$ the edges of $G_{2}$.

We say that $F$ is one-to-one if and only if

(Def. 13) $\quad F_{\mathbb{V}}$ is one-to-one and $F_{\mathbb{E}}$ is one-to-one.

We say that $F$ is directed if and only if

(Def. 14) for every objects $e, v, w$ such that $e \in \operatorname{dom}\left(F_{\mathbb{E}}\right)$ and $v, w \in \operatorname{dom}\left(F_{\mathbb{V}}\right)$ holds if $e$ joins $v$ to $w$ in $G_{1}$, then $\left(F_{\mathbb{E}}\right)(e)$ joins $\left(F_{\mathbb{V}}\right)(v)$ to $\left(F_{\mathbb{V}}\right)(w)$ in $G_{2}$.

We say that $F$ is semi-continuous if and only if

(Def. 15) for every objects $e, v, w$ such that $e \in \operatorname{dom}\left(F_{\mathbb{E}}\right)$ and $v, w \in \operatorname{dom}\left(F_{\mathbb{V}}\right)$ holds if $\left(F_{\mathbb{E}}\right)(e)$ joins $\left(F_{\mathbb{V}}\right)(v)$ and $\left(F_{\mathbb{V}}\right)(w)$ in $G_{2}$, then $e$ joins $v$ and $w$ in $G_{1}$.

We say that $F$ is continuous if and only if

(Def. 16) for every objects $\tilde{e}, v, w$ such that $v, w \in \operatorname{dom}\left(F_{\mathbb{V}}\right)$ and $\tilde{e}$ joins $\left(F_{\mathbb{V}}\right)(v)$ and $\left(F_{\mathbb{V}}\right)(w)$ in $G_{2}$ there exists an object $e$ such that $e$ joins $v$ and $w$ in $G_{1}$ and $e \in \operatorname{dom}\left(F_{\mathbb{E}}\right)$ and $\left(F_{\mathbb{E}}\right)(e)=\tilde{e}$.

We say that $F$ is semi-directed-continuous if and only if

(Def. 17) for every objects $e, v, w$ such that $e \in \operatorname{dom}\left(F_{\mathbb{E}}\right)$ and $v, w \in \operatorname{dom}\left(F_{\mathbb{V}}\right)$ holds if $\left(F_{\mathbb{E}}\right)(e)$ joins $\left(F_{\mathbb{V}}\right)(v)$ to $\left(F_{\mathbb{V}}\right)(w)$ in $G_{2}$, then $e$ joins $v$ to $w$ in $G_{1}$.

We say that $F$ is directed-continuous if and only if

(Def. 18) for every objects $\tilde{e}, v, w \operatorname{such}$ that $v, w \in \operatorname{dom}\left(F_{\mathbb{V}}\right)$ and $\tilde{e}$ joins $\left(F_{\mathbb{V}}\right)(v)$ to $\left(F_{\mathbb{V}}\right)(w)$ in $G_{2}$ there exists an object $e$ such that $e$ joins $v$ to $w$ in $G_{1}$ and $e \in \operatorname{dom}\left(F_{\mathbb{E}}\right)$ and $\left(F_{\mathbb{E}}\right)(e)=\tilde{e}$. 
Let us consider graphs $G_{1}, G_{2}$ and a partial graph mapping $F$ from $G_{1}$ to $G_{2}$. Now we state the propositions:

(13) $F$ is directed if and only if for every object $e$ such that $e \in \operatorname{dom}\left(F_{\mathbb{E}}\right)$ holds (the source of $\left.G_{2}\right)\left(\left(F_{\mathbb{E}}\right)(e)\right)=\left(F_{\mathbb{V}}\right)\left(\left(\right.\right.$ the source of $\left.\left.G_{1}\right)(e)\right)$ and (the target of $\left.G_{2}\right)\left(\left(F_{\mathbb{E}}\right)(e)\right)=\left(F_{\mathbb{V}}\right)\left(\left(\right.\right.$ the target of $\left.\left.G_{1}\right)(e)\right)$. The theorem is a consequence of (5).

(14) $\quad F$ is directed if and only if (the source of $\left.G_{2}\right) \cdot\left(F_{\mathbb{E}}\right)=\left(F_{\mathbb{V}}\right) \cdot(($ the source of $\left.G_{1}\right)\left\lceil\operatorname{dom}\left(F_{\mathbb{E}}\right)\right)$ and (the target of $\left.G_{2}\right) \cdot\left(F_{\mathbb{E}}\right)=\left(F_{\mathbb{V}}\right) \cdot(($ the target of $\left.G_{1}\right)\left\lceil\operatorname{dom}\left(F_{\mathbb{E}}\right)\right)$. The theorem is a consequence of (13) and (5).

(15) $F$ is semi-continuous if and only if for every objects $e, v, w$ such that $e \in \operatorname{dom}\left(F_{\mathbb{E}}\right)$ and $v, w \in \operatorname{dom}\left(F_{\mathbb{V}}\right)$ holds $e$ joins $v$ and $w$ in $G_{1}$ iff $\left(F_{\mathbb{E}}\right)(e)$ joins $\left(F_{\mathbb{V}}\right)(v)$ and $\left(F_{\mathbb{V}}\right)(w)$ in $G_{2}$.

(16) $F$ is semi-directed-continuous if and only if for every objects $e, v, w$ such that $e \in \operatorname{dom}\left(F_{\mathbb{E}}\right)$ and $v, w \in \operatorname{dom}\left(F_{\mathbb{V}}\right)$ holds $e$ joins $v$ to $w$ in $G_{1}$ iff $\left(F_{\mathbb{E}}\right)(e)$ joins $\left(F_{\mathbb{V}}\right)(v)$ to $\left(F_{\mathbb{V}}\right)(w)$ in $G_{2}$.

Proof: If $F$ is semi-directed-continuous, then for every objects $e, v, w$ such that $e \in \operatorname{dom}\left(F_{\mathbb{E}}\right)$ and $v, w \in \operatorname{dom}\left(F_{\mathbb{V}}\right)$ holds $e$ joins $v$ to $w$ in $G_{1}$ iff $\left(F_{\mathbb{E}}\right)(e)$ joins $\left(F_{\mathbb{V}}\right)(v)$ to $\left(F_{\mathbb{V}}\right)(w)$ in $G_{2}$.

Let $G_{1}, G_{2}$ be graphs. Note that there exists a partial graph mapping from $G_{1}$ to $G_{2}$ which is empty, one-to-one, directed-continuous, directed, continuous, semi-directed-continuous, and semi-continuous and there exists a partial graph mapping from $G_{1}$ to $G_{2}$ which is non empty, one-to-one, directed, semi-directedcontinuous, and semi-continuous.

Let $F$ be an empty partial graph mapping from $G_{1}$ to $G_{2}$. One can verify that $F_{\mathbb{V}}$ is empty as a set and $F_{\mathbb{E}}$ is empty as a set.

Let $F$ be a non empty partial graph mapping from $G_{1}$ to $G_{2}$. One can verify that $F_{\mathbb{V}}$ is non empty as a set.

Let $F$ be a one-to-one partial graph mapping from $G_{1}$ to $G_{2}$. One can verify that $F_{\mathbb{V}}$ is one-to-one as a function and $F_{\mathbb{E}}$ is one-to-one as a function.

Now we state the propositions:

(17) Let us consider graphs $G_{1}, G_{2}$, and a partial graph mapping $F$ from $G_{1}$ to $G_{2}$. If $F_{\mathbb{V}}$ is one-to-one, then $F$ is semi-continuous. The theorem is a consequence of (5) and (4).

(18) Let us consider graphs $G_{1}, G_{2}$, and a directed partial graph mapping $F$ from $G_{1}$ to $G_{2}$. If $F_{\mathbb{V}}$ is one-to-one, then $F$ is semi-directed-continuous. The theorem is a consequence of (5).

(19) Let us consider graphs $G_{1}, G_{2}$, and a semi-continuous partial graph mapping $F$ from $G_{1}$ to $G_{2}$. Suppose $\operatorname{rng} F_{\mathbb{E}}=$ the edges of $G_{2}$. Then $F$ is continuous. 
(20) Let us consider graphs $G_{1}, G_{2}$, and a semi-directed-continuous partial graph mapping $F$ from $G_{1}$ to $G_{2}$. Suppose $\operatorname{rng} F_{\mathbb{E}}=$ the edges of $G_{2}$. Then $F$ is directed-continuous.

(21) Let us consider graphs $G_{1}, G_{2}$, and a partial graph mapping $F$ from $G_{1}$ to $G_{2}$. Suppose $F_{\mathbb{V}}$ is one-to-one and $\operatorname{rng} F_{\mathbb{E}}=$ the edges of $G_{2}$. Then $F$ is continuous. The theorem is a consequence of (17) and (19).

(22) Let us consider graphs $G_{1}, G_{2}$, and a directed partial graph mapping $F$ from $G_{1}$ to $G_{2}$. Suppose $F_{\mathbb{V}}$ is one-to-one and $\operatorname{rng} F_{\mathbb{E}}=$ the edges of $G_{2}$. Then $F$ is directed-continuous. The theorem is a consequence of (18) and (20).

(23) Let us consider graphs $G_{1}, G_{2}$, and a continuous partial graph mapping $F$ from $G_{1}$ to $G_{2}$. If $F_{\mathbb{E}}$ is one-to-one, then $F$ is semi-continuous.

Let us consider graphs $G_{1}, G_{2}$ and a directed-continuous partial graph mapping $F$ from $G_{1}$ to $G_{2}$. Now we state the propositions:

(24) If $F_{\mathbb{E}}$ is one-to-one, then $F$ is semi-directed-continuous.

(25) If $F_{\mathbb{E}}$ is one-to-one, then $F$ is directed. The theorem is a consequence of (4).

(26) Let us consider graphs $G_{1}, G_{2}$, a semi-continuous partial graph mapping $F$ from $G_{1}$ to $G_{2}$, and objects $v_{1}, v_{2}$. Suppose $v_{1}, v_{2} \in \operatorname{dom}\left(F_{\mathbb{V}}\right)$ and $\left(F_{\mathbb{V}}\right)\left(v_{1}\right)=\left(F_{\mathbb{V}}\right)\left(v_{2}\right)$ and there exist objects $e, w$ such that $e \in \operatorname{dom}\left(F_{\mathbb{E}}\right)$ and $w \in \operatorname{dom}\left(F_{\mathbb{V}}\right)$ and $\left(F_{\mathbb{E}}\right)(e)$ joins $\left(F_{\mathbb{V}}\right)\left(v_{1}\right)$ and $\left(F_{\mathbb{V}}\right)(w)$ in $G_{2}$. Then $v_{1}=v_{2}$.

(27) Let us consider graphs $G_{1}, G_{2}$, and a semi-continuous partial graph mapping $F$ from $G_{1}$ to $G_{2}$. Suppose for every object $v$ such that $v \in$ $\operatorname{dom}\left(F_{\mathbb{V}}\right)$ there exist objects $e, w$ such that $e \in \operatorname{dom}\left(F_{\mathbb{E}}\right)$ and $w \in \operatorname{dom}\left(F_{\mathbb{V}}\right)$ and $\left(F_{\mathbb{E}}\right)(e)$ joins $\left(F_{\mathbb{V}}\right)(v)$ and $\left(F_{\mathbb{V}}\right)(w)$ in $G_{2}$. Then $F_{\mathbb{V}}$ is one-to-one. The theorem is a consequence of $(26)$.

(28) Let us consider graphs $G_{1}, G_{2}$, a semi-directed-continuous partial graph mapping $F$ from $G_{1}$ to $G_{2}$, and objects $v_{1}, v_{2}$. Suppose $v_{1}, v_{2} \in \operatorname{dom}\left(F_{\mathbb{V}}\right)$ and $\left(F_{\mathbb{V}}\right)\left(v_{1}\right)=\left(F_{\mathbb{V}}\right)\left(v_{2}\right)$ and there exist objects $e$, w such that $e \in$ $\operatorname{dom}\left(F_{\mathbb{E}}\right)$ and $w \in \operatorname{dom}\left(F_{\mathbb{V}}\right)$ and $\left(F_{\mathbb{E}}\right)(e)$ joins $\left(F_{\mathbb{V}}\right)\left(v_{1}\right)$ to $\left(F_{\mathbb{V}}\right)(w)$ in $G_{2}$. Then $v_{1}=v_{2}$.

(29) Let us consider graphs $G_{1}, G_{2}$, and a semi-directed-continuous partial graph mapping $F$ from $G_{1}$ to $G_{2}$. Suppose for every object $v$ such that $v \in$ $\operatorname{dom}\left(F_{\mathbb{V}}\right)$ there exist objects $e, w$ such that $e \in \operatorname{dom}\left(F_{\mathbb{E}}\right)$ and $w \in \operatorname{dom}\left(F_{\mathbb{V}}\right)$ and $\left(F_{\mathbb{E}}\right)(e)$ joins $\left(F_{\mathbb{V}}\right)(v)$ to $\left(F_{\mathbb{V}}\right)(w)$ in $G_{2}$. Then $F_{\mathbb{V}}$ is one-to-one. The theorem is a consequence of $(28)$.

Let $G_{1}, G_{2}$ be graphs. One can verify that every partial graph mapping from 
$G_{1}$ to $G_{2}$ which is one-to-one is also semi-continuous and every partial graph mapping from $G_{1}$ to $G_{2}$ which is one-to-one and directed is also semi-directedcontinuous and every partial graph mapping from $G_{1}$ to $G_{2}$ which is one-to-one and onto is also continuous and every partial graph mapping from $G_{1}$ to $G_{2}$ which is directed, one-to-one, and onto is also directed-continuous.

Every partial graph mapping from $G_{1}$ to $G_{2}$ which is semi-continuous and onto is also continuous and every partial graph mapping from $G_{1}$ to $G_{2}$ which is semi-directed-continuous is also directed and semi-continuous and every partial graph mapping from $G_{1}$ to $G_{2}$ which is semi-directed-continuous and onto is also directed-continuous and every partial graph mapping from $G_{1}$ to $G_{2}$ which is directed-continuous is also continuous.

Every partial graph mapping from $G_{1}$ to $G_{2}$ which is directed-continuous and one-to-one is also directed and semi-directed-continuous and every partial graph mapping from $G_{1}$ to $G_{2}$ which is empty is also one-to-one, directed-continuous, directed, and continuous and every partial graph mapping from $G_{1}$ to $G_{2}$ which is total is also non empty and every partial graph mapping from $G_{1}$ to $G_{2}$ which is onto is also non empty.

Let $G$ be a graph. One can verify that $\mathrm{id}_{G}$ is total, non empty, onto, one-toone, and directed-continuous.

Let us consider graphs $G_{1}, G_{2}$, a partial function $f$ from the vertices of $G_{1}$ to the vertices of $G_{2}$, and a partial function $g$ from the edges of $G_{1}$ to the edges of $G_{2}$. Now we state the propositions:

(30) Suppose for every object $e$ such that $e \in \operatorname{dom} g$ holds (the source of $\left.G_{1}\right)(e)$, (the target of $\left.G_{1}\right)(e) \in \operatorname{dom} f$ and for every objects $e, v, w$ such that $e \in \operatorname{dom} g$ and $v, w \in \operatorname{dom} f$ holds if $e$ joins $v$ to $w$ in $G_{1}$, then $g(e)$ joins $f(v)$ to $f(w)$ in $G_{2}$. Then $\langle f, g\rangle$ is a directed partial graph mapping from $G_{1}$ to $G_{2}$. The theorem is a consequence of (8).

(31) Suppose for every object $e$ such that $e \in \operatorname{dom} g$ holds (the source of $\left.G_{1}\right)(e)$, (the target of $\left.G_{1}\right)(e) \in \operatorname{dom} f$ and for every objects $e, v, w$ such that $e \in \operatorname{dom} g$ and $v, w \in \operatorname{dom} f$ holds $e$ joins $v$ and $w$ in $G_{1}$ iff $g(e)$ joins $f(v)$ and $f(w)$ in $G_{2}$. Then $\langle f, g\rangle$ is a semi-continuous partial graph mapping from $G_{1}$ to $G_{2}$. The theorem is a consequence of (8).

(32) Suppose for every object $e$ such that $e \in \operatorname{dom} g$ holds (the source of $\left.G_{1}\right)(e)$, (the target of $\left.G_{1}\right)(e) \in \operatorname{dom} f$ and for every objects $e, v, w$ such that $e \in \operatorname{dom} g$ and $v, w \in \operatorname{dom} f$ holds $e$ joins $v$ to $w$ in $G_{1}$ iff $g(e)$ joins $f(v)$ to $f(w)$ in $G_{2}$. Then $\langle f, g\rangle$ is a semi-directed-continuous partial graph mapping from $G_{1}$ to $G_{2}$. The theorem is a consequence of (8).

(33) Let us consider graphs $G_{1}, G_{2}$. Then $\langle\emptyset, \emptyset\rangle$ is an empty, one-to-one, directed-continuous partial graph mapping from $G_{1}$ to $G_{2}$. 
(34) Let us consider graphs $G_{1}, G_{2}$, and a partial graph mapping $F$ from $G_{1}$ to $G_{2}$. Suppose $F$ is total. Let us consider a vertex $v$ of $G_{1}$. Then $\left(F_{\mathbb{V}}\right)(v)$ is a vertex of $G_{2}$.

(35) Let us consider graphs $G_{1}, G_{2}$, and a partial graph mapping $F$ from $G_{1}$ to $G_{2}$. Suppose $F$ is total. Then

(i) if $G_{2}$ is loopless, then $G_{1}$ is loopless, and

(ii) if $G_{2}$ is edgeless, then $G_{1}$ is edgeless.

The theorem is a consequence of (4).

(36) Let us consider graphs $G_{1}, G_{2}$, and a continuous partial graph mapping $F$ from $G_{1}$ to $G_{2}$. Suppose $\operatorname{rng} F_{\mathbb{V}}=$ the vertices of $G_{2}$. If $G_{1}$ is loopless, then $G_{2}$ is loopless.

Proof: For every object $v$, there exists no object $e$ such that $e$ joins $v$ and $v$ in $G_{2}$.

(37) Let us consider graphs $G_{1}, G_{2}$, and a semi-continuous partial graph mapping $F$ from $G_{1}$ to $G_{2}$. If $F$ is onto, then if $G_{1}$ is loopless, then $G_{2}$ is loopless.

Let us consider graphs $G_{1}, G_{2}$ and a partial graph mapping $F$ from $G_{1}$ to $G_{2}$. Now we state the propositions:

(38) If $\operatorname{rng} F_{\mathbb{E}}=$ the edges of $G_{2}$, then if $G_{1}$ is edgeless, then $G_{2}$ is edgeless.

(39) If $F$ is onto, then if $G_{1}$ is edgeless, then $G_{2}$ is edgeless.

(40) Let us consider a graph $G_{1}$, a non-multi graph $G_{2}$, and partial graph mappings $F_{1}, F_{2}$ from $G_{1}$ to $G_{2}$. Suppose $F_{1 \mathbb{V}}=F_{2 \mathbb{V}}$ and $\operatorname{dom}\left(F_{1 \mathbb{E}}\right)=$ $\operatorname{dom}\left(F_{2 \mathbb{E}}\right)$. Then $F_{1}=F_{2}$. The theorem is a consequence of (5) and (4).

(41) Let us consider a graph $G_{1}$, a non-directed-multi graph $G_{2}$, and directed partial graph mappings $F_{1}, F_{2}$ from $G_{1}$ to $G_{2}$. Suppose $F_{1 \mathbb{V}}=F_{2 \mathbb{V}}$ and $\operatorname{dom}\left(F_{1 \mathbb{E}}\right)=\operatorname{dom}\left(F_{2 \mathbb{E}}\right)$. Then $F_{1}=F_{2}$. The theorem is a consequence of (5).

(42) Let us consider a non-multi graph $G_{1}$, a graph $G_{2}$, and a semi-continuous partial graph mapping $F$ from $G_{1}$ to $G_{2}$. Then $F_{\mathbb{E}}$ is one-to-one. The theorem is a consequence of (5) and (4).

(43) Let us consider a non-multi graph $G_{1}$, a graph $G_{2}$, and a partial graph mapping $F$ from $G_{1}$ to $G_{2}$. If $F_{\mathbb{V}}$ is one-to-one, then $F_{\mathbb{E}}$ is one-to-one. The theorem is a consequence of (5) and (4).

(44) Let us consider a non-directed-multi graph $G_{1}$, a graph $G_{2}$, and a directed partial graph mapping $F$ from $G_{1}$ to $G_{2}$. If $F_{\mathbb{V}}$ is one-to-one, then $F_{\mathbb{E}}$ is one-to-one. The theorem is a consequence of (5).

Let $G_{1}$ be a graph and $G_{2}$ be a loopless graph. Observe that every partial graph mapping from $G_{1}$ to $G_{2}$ which is directed and semi-continuous is also semi- 
directed-continuous and every partial graph mapping from $G_{1}$ to $G_{2}$ which is directed and continuous is also directed-continuous.

Let $G_{1}$ be a trivial graph and $G_{2}$ be a graph. Observe that every partial graph mapping from $G_{1}$ to $G_{2}$ is directed and every partial graph mapping from $G_{1}$ to $G_{2}$ which is semi-continuous is also semi-directed-continuous and every partial graph mapping from $G_{1}$ to $G_{2}$ which is continuous is also directed-continuous.

Let $G_{1}$ be a trivial, non-directed-multi graph. Note that every partial graph mapping from $G_{1}$ to $G_{2}$ is one-to-one.

Let $G_{1}$ be a trivial, edgeless graph. Observe that every partial graph mapping from $G_{1}$ to $G_{2}$ which is non empty is also total.

Let $G_{1}$ be a graph and $G_{2}$ be a trivial, edgeless graph. Note that every partial graph mapping from $G_{1}$ to $G_{2}$ which is non empty is also onto and every partial graph mapping from $G_{1}$ to $G_{2}$ is semi-continuous and continuous.

Let $G_{1}, G_{2}$ be graphs and $F$ be a partial graph mapping from $G_{1}$ to $G_{2}$. We say that $F$ is weak subgraph embedding if and only if

(Def. 19) $F$ is total and one-to-one.

We say that $F$ is strong subgraph embedding if and only if

(Def. 20) $F$ is total, one-to-one, and continuous.

We say that $F$ is isomorphism if and only if

(Def. 21) $F$ is total, one-to-one, and onto.

We say that $F$ is directed-isomorphism if and only if

(Def. 22) $F$ is directed, total, one-to-one, and onto.

One can check that every partial graph mapping from $G_{1}$ to $G_{2}$ which is weak subgraph embedding is also total, non empty, one-to-one, and semi-continuous and every partial graph mapping from $G_{1}$ to $G_{2}$ which is total and one-to-one is also weak subgraph embedding and every partial graph mapping from $G_{1}$ to $G_{2}$ which is strong subgraph embedding is also total, non empty, one-to-one, continuous, and weak subgraph embedding and every partial graph mapping from $G_{1}$ to $G_{2}$ which is total, one-to-one, and continuous is also strong subgraph embedding.

Every partial graph mapping from $G_{1}$ to $G_{2}$ which is weak subgraph embedding and continuous is also strong subgraph embedding and every partial graph mapping from $G_{1}$ to $G_{2}$ which is isomorphism is also onto, semi-continuous, continuous, total, non empty, one-to-one, weak subgraph embedding, and strong subgraph embedding and every partial graph mapping from $G_{1}$ to $G_{2}$ which is total, one-to-one, onto, and continuous is also isomorphism and every partial graph mapping from $G_{1}$ to $G_{2}$ which is strong subgraph embedding and onto is also isomorphism. 
Every partial graph mapping from $G_{1}$ to $G_{2}$ which is weak subgraph embedding, continuous, and onto is also isomorphism and every partial graph mapping from $G_{1}$ to $G_{2}$ which is directed-isomorphism is also directed, isomorphism, continuous, total, non empty, semi-directed-continuous, semi-continuous, oneto-one, weak subgraph embedding, and strong subgraph embedding and every partial graph mapping from $G_{1}$ to $G_{2}$ which is directed and isomorphism is also directed-continuous and directed-isomorphism.

Let $G$ be a graph. Let us note that $\operatorname{id}_{G}$ is weak subgraph embedding, strong subgraph embedding, isomorphism, and directed-isomorphism and there exists a partial graph mapping from $G$ to $G$ which is weak subgraph embedding, strong subgraph embedding, isomorphism, and directed-isomorphism.

Now we state the propositions:

(45) Let us consider graphs $G_{1}, G_{2}$, and a partial graph mapping $F$ from $G_{1}$ to $G_{2}$. Suppose $F$ is weak subgraph embedding. Then

(i) $G_{1} \cdot \operatorname{order}() \subseteq G_{2} \cdot \operatorname{order}()$, and

(ii) $G_{1} \cdot \operatorname{size}() \subseteq G_{2} \cdot \operatorname{size}()$.

(46) Let us consider graphs $G_{1}, G_{2}$, a partial graph mapping $F$ from $G_{1}$ to $G_{2}$, and subsets $X, Y$ of the vertices of $G_{1}$. Suppose $F$ is weak subgraph embed-

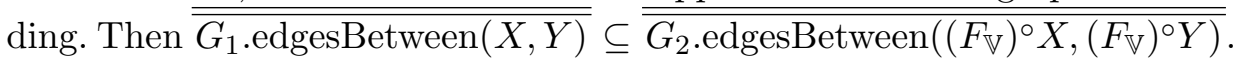
Proof: Set $f=F_{\mathbb{E}}\left\lceil G_{1}\right.$.edgesBetween $(X, Y)$. For every object $y$ such that $y \in \operatorname{rng} f$ holds $y \in G_{2}$.edgesBetween $\left(\left(F_{\mathbb{V}}\right)^{\circ} X,\left(F_{\mathbb{V}}\right)^{\circ} Y\right)$.

(47) Let us consider graphs $G_{1}, G_{2}$, a partial graph mapping $F$ from $G_{1}$ to $G_{2}$, and a subset $X$ of the vertices of $G_{1}$. Suppose $F$ is weak subgraph embedding. Then $\overline{\overline{G_{1} \text {.edgesBetween }(X)}} \subseteq \overline{\overline{G_{2} \text {.edgesBetween }\left(\left(F_{\mathbb{V}}\right)^{\circ} X\right)}}$.

Proof: Set $f=F_{\mathbb{E}}\left\lceil G_{1}\right.$.edgesBetween $(X)$. For every object $y$ such that $y \in \operatorname{rng} f$ holds $y \in G_{2}$.edgesBetween $\left(\left(F_{\mathbb{V}}\right)^{\circ} X\right)$.

(48) Let us consider graphs $G_{1}, G_{2}$, a directed partial graph mapping $F$ from $G_{1}$ to $G_{2}$, and subsets $X, Y$ of the vertices of $G_{1}$. Suppose $F$ is weak subgraph embedding. Then $\overline{\overline{G_{1} \text {.edgesDBetween }(X, Y)} \subseteq}$

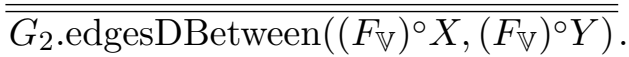

Proof: Set $f=F_{\mathbb{E}}\left\lceil G_{1}\right.$.edgesDBetween $(X, Y)$. For every object $y$ such that $y \in \operatorname{rng} f$ holds $y \in G_{2}$.edgesDBetween $\left(\left(F_{\mathbb{V}}\right)^{\circ} X,\left(F_{\mathbb{V}}\right)^{\circ} Y\right)$.

Let us consider graphs $G_{1}, G_{2}$ and a partial graph mapping $F$ from $G_{1}$ to $G_{2}$. Now we state the propositions:

(49) Suppose $F$ is weak subgraph embedding. Then

(i) if $G_{2}$ is trivial, then $G_{1}$ is trivial, and

(ii) if $G_{2}$ is non-multi, then $G_{1}$ is non-multi, and 
(iii) if $G_{2}$ is simple, then $G_{1}$ is simple, and

(iv) if $G_{2}$ is finite, then $G_{1}$ is finite.

Proof: If $G_{2}$ is non-multi, then $G_{1}$ is non-multi. $G_{1}$.order ()$\subseteq G_{2}$.order() and $G_{1} \cdot \operatorname{size}() \subseteq G_{2} \cdot \operatorname{size}()$.

(50) Suppose $F$ is directed and weak subgraph embedding. Then

(i) if $G_{2}$ is non-directed-multi, then $G_{1}$ is non-directed-multi, and

(ii) if $G_{2}$ is directed-simple, then $G_{1}$ is directed-simple.

Proof: If $G_{2}$ is non-directed-multi, then $G_{1}$ is non-directed-multi. $G_{1}$ is loopless and non-directed-multi.

(51) Let us consider finite graphs $G_{1}, G_{2}$, and a partial graph mapping $F$ from $G_{1}$ to $G_{2}$. Suppose $F$ is strong subgraph embedding and $G_{1}$.order ()$=$ $G_{2}$.order() and $G_{1} \cdot \operatorname{size}()=G_{2} \cdot \operatorname{size}()$. Then $F$ is isomorphism.

(52) Let us consider graphs $G_{1}, G_{2}$, and a partial graph mapping $F$ from $G_{1}$ to $G_{2}$. Suppose $F$ is strong subgraph embedding. If $G_{2}$ is complete, then $G_{1}$ is complete.

Let $G_{1}, G_{2}$ be graphs. We say that $G_{2}$ is $G_{1}$-isomorphic if and only if

(Def. 23) there exists a partial graph mapping $F$ from $G_{1}$ to $G_{2}$ such that $F$ is isomorphism.

We say that $G_{2}$ is $G_{1}$-directed-isomorphic if and only if

(Def. 24) there exists a partial graph mapping $F$ from $G_{1}$ to $G_{2}$ such that $F$ is directed-isomorphism.

Let $G$ be a graph. Note that every graph which is $G$-directed-isomorphic is also $G$-isomorphic and there exists a graph which is $G$-directed-isomorphic and $G$-isomorphic.

Now we state the proposition:

(53) Every graph is directed-isomorphic and isomorphic to itself.

Let $G_{1}$ be a graph and $G_{2}$ be a $G_{1}$-isomorphic graph. Let us observe that there exists a partial graph mapping from $G_{1}$ to $G_{2}$ which is isomorphism, strong subgraph embedding, weak subgraph embedding, total, non empty, one-to-one, onto, semi-continuous, and continuous.

An isomorphism between $G_{1}$ and $G_{2}$ is an isomorphism partial graph mapping from $G_{1}$ to $G_{2}$. Let $G_{2}$ be a $G_{1}$-directed-isomorphic graph. One can verify that there exists a partial graph mapping from $G_{1}$ to $G_{2}$ which is isomorphism, strong subgraph embedding, weak subgraph embedding, total, non empty, oneto-one, onto, directed, semi-directed-continuous, and directed-continuous.

A directed isomorphism of $G_{1}$ and $G_{2}$ is a directed-isomorphism partial graph mapping from $G_{1}$ to $G_{2}$. Let $G_{1}, G_{2}$ be w-graphs and $F$ be a partial graph mapping from $G_{1}$ to $G_{2}$. We say that $F$ preserves weight if and only if 
(Def. 25) (the weight of $\left.G_{2}\right) \cdot\left(F_{\mathbb{E}}\right)=\left(\right.$ the weight of $\left.G_{1}\right)\left\lceil\operatorname{dom}\left(F_{\mathbb{E}}\right)\right.$.

Let $G_{1}, G_{2}$ be e-graphs. We say that $F$ preserves elabel if and only if

(Def. 26) (the elabel of $\left.G_{2}\right) \cdot\left(F_{\mathbb{E}}\right)=\left(\right.$ the elabel of $\left.G_{1}\right)\left\lceil\operatorname{dom}\left(F_{\mathbb{E}}\right)\right.$.

Let $G_{1}, G_{2}$ be v-graphs. We say that $F$ preserves vlabel if and only if

(Def. 27) (the vlabel of $\left.G_{2}\right) \cdot\left(F_{\mathbb{V}}\right)=\left(\right.$ the vlabel of $\left.G_{1}\right)\left\lceil\operatorname{dom}\left(F_{\mathbb{V}}\right)\right.$.

Let $G_{1}, G_{2}$ be ordered graphs. We say that $F$ preserves ordering if and only if

(Def. 28) (the ordering of $\left.G_{2}\right) \cdot\left(F_{\mathbb{V}}\right)=$ the ordering of $G_{1}\left\lceil\operatorname{dom}\left(F_{\mathbb{V}}\right)\right.$.

Let $G$ be a w-graph. Note that $\operatorname{id}_{G}$ preserves weight.

Let $G$ be an e-graph. Let us note that $\operatorname{id}_{G}$ preserves elabel.

Let $G$ be a v-graph. Observe that $\mathrm{id}_{G}$ preserves vlabel.

Let $G$ be an ordered graph. Let us observe that $\operatorname{id}_{G}$ preserves ordering.

Let $G_{1}, G_{2}$ be graphs and $F$ be a partial graph mapping from $G_{1}$ to $G_{2}$. The functor $\operatorname{dom} F$ yielding a subgraph of $G_{1}$ induced by $\operatorname{dom}\left(F_{\mathbb{V}}\right)$ and $\operatorname{dom}\left(F_{\mathbb{E}}\right)$ is defined by the term

(Def. 29) the plain subgraph of $G_{1}$ induced by $\operatorname{dom}\left(F_{\mathbb{V}}\right)$ and $\operatorname{dom}\left(F_{\mathbb{E}}\right)$.

The functor $\operatorname{rng} F$ yielding a subgraph of $G_{2}$ induced by $\operatorname{rng} F_{\mathbb{V}}$ and $\operatorname{rng} F_{\mathbb{E}}$ is defined by the term

(Def. 30) the plain subgraph of $G_{2}$ induced by $\operatorname{rng} F_{\mathbb{V}}$ and $\operatorname{rng} F_{\mathbb{E}}$.

One can verify that $\operatorname{dom} F$ is plain and $\operatorname{rng} F$ is plain.

Let us consider graphs $G_{1}, G_{2}$ and a non empty partial graph mapping $F$ from $G_{1}$ to $G_{2}$. Now we state the propositions:

(i) the vertices of $\operatorname{dom} F=\operatorname{dom}\left(F_{\mathbb{V}}\right)$, and

(ii) the edges of $\operatorname{dom} F=\operatorname{dom}\left(F_{\mathbb{E}}\right)$, and

(iii) the vertices of $\operatorname{rng} F=\operatorname{rng} F_{\mathbb{V}}$, and

(iv) the edges of $\operatorname{rng} F=\operatorname{rng} F_{\mathbb{E}}$.

The theorem is a consequence of $(7)$.

(55) $F$ is total if and only if $\operatorname{dom} F \approx G_{1}$. The theorem is a consequence of (54).

(56) $F$ is onto if and only if $\operatorname{rng} F \approx G_{2}$. The theorem is a consequence of (54).

Let $G_{1}, G_{2}$ be graphs, $H$ be a subgraph of $G_{1}$, and $F$ be a partial graph mapping from $G_{1}$ to $G_{2}$. The functor $F\lceil H$ yielding a partial graph mapping from $H$ to $G_{2}$ is defined by the term

(Def. 31) $\left\langle F_{\mathbb{V}} \uparrow(\right.$ the vertices of $H), F_{\mathbb{E}} \uparrow($ the edges of $\left.H)\right\rangle$.

Now we state the propositions: 
(57) Let us consider graphs $G_{1}, G_{2}$, a subgraph $H$ of $G_{1}$, and a partial graph mapping $F$ from $G_{1}$ to $G_{2}$. Then

(i) if $F$ is empty, then $F\lceil H$ is empty, and

(ii) if $F$ is total, then $F\lceil H$ is total, and

(iii) if $F$ is one-to-one, then $F\lceil H$ is one-to-one, and

(iv) if $F$ is weak subgraph embedding, then $F\lceil H$ is weak subgraph embedding, and

(v) if $F$ is semi-continuous, then $F\lceil H$ is semi-continuous, and

(vi) if $F$ is not onto, then $F\lceil H$ is not onto, and

(vii) if $F$ is directed, then $F\lceil H$ is directed, and

(viii) if $F$ is semi-directed-continuous, then $F\lceil H$ is semi-directed-continuous.

Proof: If $F$ is total, then $F\lceil H$ is total. If $F$ is semi-continuous, then $F\lceil H$ is semi-continuous. If $F\lceil H$ is onto, then $F$ is onto. If $F$ is directed, then $F\lceil H$ is directed. If $F$ is semi-directed-continuous, then $F\lceil H$ is semidirected-continuous.

(58) Let us consider graphs $G_{1}, G_{2}$, a set $V$, a subgraph $H$ of $G_{1}$ induced by $V$, and a partial graph mapping $F$ from $G_{1}$ to $G_{2}$. Then

(i) if $F$ is continuous, then $F\lceil H$ is continuous, and

(ii) if $F$ is strong subgraph embedding, then $F\lceil H$ is strong subgraph embedding, and

(iii) if $F$ is directed-continuous, then $F\lceil H$ is directed-continuous.

The theorem is a consequence of (57).

Let $G_{1}, G_{2}$ be graphs, $H$ be a subgraph of $G_{1}$, and $F$ be an empty partial graph mapping from $G_{1}$ to $G_{2}$. Let us observe that $F\lceil H$ is empty.

Let $F$ be a one-to-one partial graph mapping from $G_{1}$ to $G_{2}$. Let us observe that $F\lceil H$ is one-to-one.

Let $F$ be a semi-continuous partial graph mapping from $G_{1}$ to $G_{2}$. Observe that $F\lceil H$ is semi-continuous.

Let $V$ be a set, $H$ be a subgraph of $G_{1}$ induced by $V$, and $F$ be a continuous partial graph mapping from $G_{1}$ to $G_{2}$. Let us observe that $F\lceil H$ is continuous.

Let $H$ be a subgraph of $G_{1}$ and $F$ be a directed partial graph mapping from $G_{1}$ to $G_{2}$. Note that $F\lceil H$ is directed.

Let $F$ be a semi-directed-continuous partial graph mapping from $G_{1}$ to $G_{2}$. One can check that $F\lceil H$ is semi-directed-continuous.

Let $V$ be a set, $H$ be a subgraph of $G_{1}$ induced by $V$, and $F$ be a directedcontinuous partial graph mapping from $G_{1}$ to $G_{2}$. Note that $F\lceil H$ is directedcontinuous. 
Let $F$ be a non empty partial graph mapping from $G_{1}$ to $G_{2}$. One can verify that $F \uparrow \operatorname{dom} F$ is total.

Now we state the propositions:

(59) Let us consider graphs $G_{1}, G_{2}$, a subgraph $H$ of $G_{1}$, and a partial graph mapping $F$ from $G_{1}$ to $G_{2}$. Then

(i) $\operatorname{dom}\left(\left(F\lceil H)_{\mathbb{V}}\right)=\operatorname{dom}\left(F_{\mathbb{V}}\right) \cap(\right.$ the vertices of $H)$, and

(ii) $\operatorname{dom}\left(\left(F\lceil H)_{\mathbb{E}}\right)=\operatorname{dom}\left(F_{\mathbb{E}}\right) \cap(\right.$ the edges of $H)$.

(60) Let us consider w-graphs $G_{1}, G_{2}$, a w-subgraph $H$ of $G_{1}$, and a partial graph mapping $F$ from $G_{1}$ to $G_{2}$. If $F$ preserves weight, then $F\lceil H$ preserves weight. The theorem is a consequence of (59).

(61) Let us consider e-graphs $G_{1}, G_{2}$, an e-subgraph $H$ of $G_{1}$, and a partial graph mapping $F$ from $G_{1}$ to $G_{2}$. If $F$ preserves elabel, then $F \nmid H$ preserves elabel. The theorem is a consequence of (59).

(62) Let us consider v-graphs $G_{1}, G_{2}$, a v-subgraph $H$ of $G_{1}$, and a partial graph mapping $F$ from $G_{1}$ to $G_{2}$. If $F$ preserves vlabel, then $F\lceil H$ preserves vlabel. The theorem is a consequence of (59).

Let $G_{1}, G_{2}$ be graphs, $H$ be a subgraph of $G_{2}$, and $F$ be a partial graph mapping from $G_{1}$ to $G_{2}$. The functor $H 1 F$ yielding a partial graph mapping from $G_{1}$ to $H$ is defined by the term

(Def. 32) $\left\langle(\right.$ the vertices of $H) 1 F_{\mathbb{V}}$, (the edges of $\left.\left.H\right) 1 F_{\mathbb{E}}\right\rangle$.

Now we state the proposition:

(63) Let us consider graphs $G_{1}, G_{2}$, a subgraph $H$ of $G_{2}$, and a partial graph mapping $F$ from $G_{1}$ to $G_{2}$. Then

(i) if $F$ is empty, then $H 1 F$ is empty, and

(ii) if $F$ is one-to-one, then $H 1 F$ is one-to-one, and

(iii) if $F$ is onto, then $H 1 F$ is onto, and

(iv) if $F$ is not total, then $H 1 F$ is not total, and

(v) if $F$ is directed, then $H \uparrow F$ is directed, and

(vi) if $F$ is semi-continuous, then $H 1 F$ is semi-continuous, and

(vii) if $F$ is continuous, then $H 1 F$ is continuous, and

(viii) if $F$ is semi-directed-continuous, then $H 1 F$ is semi-directed-continuous, and

(ix) if $F$ is directed-continuous, then $H 1 F$ is directed-continuous.

Proof: If $F$ is onto, then $H 1 F$ is onto. If $F$ is directed, then $H 1 F$ is directed. If $F$ is semi-continuous, then $H 1 F$ is semi-continuous. If $F$ is continuous, then $H 1 F$ is continuous. If $F$ is semi-directed-continuous, then 
$H 1 F$ is semi-directed-continuous. If $F$ is directed-continuous, then $H 1 F$ is directed-continuous.

Let $G_{1}, G_{2}$ be graphs, $H$ be a subgraph of $G_{2}$, and $F$ be an empty partial graph mapping from $G_{1}$ to $G_{2}$. One can verify that $H 1 F$ is empty.

Let $F$ be a one-to-one partial graph mapping from $G_{1}$ to $G_{2}$. Let us observe that $H 1 F$ is one-to-one.

Let $F$ be a semi-continuous partial graph mapping from $G_{1}$ to $G_{2}$. Observe that $H 1 F$ is semi-continuous.

Let $F$ be a continuous partial graph mapping from $G_{1}$ to $G_{2}$. Let us note that $H 1 F$ is continuous.

Let $F$ be a directed partial graph mapping from $G_{1}$ to $G_{2}$. Note that $H 1 F$ is directed.

Let $F$ be a semi-directed-continuous partial graph mapping from $G_{1}$ to $G_{2}$. One can check that $H 1 F$ is semi-directed-continuous.

Let $F$ be a directed-continuous partial graph mapping from $G_{1}$ to $G_{2}$. One can verify that $H 1 F$ is directed-continuous.

Let $F$ be a non empty partial graph mapping from $G_{1}$ to $G_{2}$. Observe that $\operatorname{rng} F 1 F$ is onto.

Now we state the propositions:

(64) Let us consider graphs $G_{1}, G_{2}$, a subgraph $H$ of $G_{2}$, and a partial graph mapping $F$ from $G_{1}$ to $G_{2}$. Then

(i) $\operatorname{rng}(H 1 F)_{\mathbb{V}}=\operatorname{rng} F_{\mathbb{V}} \cap($ the vertices of $H)$, and

(ii) $\operatorname{rng}(H 1 F)_{\mathbb{E}}=\operatorname{rng} F_{\mathbb{E}} \cap($ the edges of $H)$.

(65) Let us consider w-graphs $G_{1}, G_{2}$, a w-subgraph $H$ of $G_{2}$, and a partial graph mapping $F$ from $G_{1}$ to $G_{2}$. If $F$ preserves weight, then $H 1 F$ preserves weight.

(66) Let us consider e-graphs $G_{1}, G_{2}$, an e-subgraph $H$ of $G_{2}$, and a partial graph mapping $F$ from $G_{1}$ to $G_{2}$. If $F$ preserves elabel, then $H 1 F$ preserves elabel.

(67) Let us consider v-graphs $G_{1}, G_{2}$, a v-subgraph $H$ of $G_{2}$, and a partial graph mapping $F$ from $G_{1}$ to $G_{2}$. If $F$ preserves vlabel, then $H 1 F$ preserves vlabel.

(68) Let us consider graphs $G_{1}, G_{2}$, a partial graph mapping $F$ from $G_{1}$ to $G_{2}$, a subgraph $H_{1}$ of $G_{1}$, and a subgraph $H_{2}$ of $G_{2}$. Then $\left(H_{2} \uparrow F\right)\left\lceil H_{1}=\right.$ $H_{2} \uparrow\left(F\left\lceil H_{1}\right)\right.$.

Let $G_{1}, G_{2}$ be graphs and $F$ be a one-to-one partial graph mapping from $G_{1}$ to $G_{2}$. The functor $F^{-1}$ yielding a partial graph mapping from $G_{2}$ to $G_{1}$ is defined by the term 
(Def. 33) $\left\langle\left(F_{\mathbb{V}}\right)^{-1},\left(F_{\mathbb{E}}\right)^{-1}\right\rangle$.

One can verify that $F^{-1}$ is one-to-one and semi-continuous.

Let $F$ be an empty, one-to-one partial graph mapping from $G_{1}$ to $G_{2}$. One can verify that $F^{-1}$ is empty.

Let $F$ be a non empty, one-to-one partial graph mapping from $G_{1}$ to $G_{2}$. Let us note that $F^{-1}$ is non empty.

Let $F$ be a one-to-one, semi-directed-continuous partial graph mapping from $G_{1}$ to $G_{2}$. One can verify that $F^{-1}$ is semi-directed-continuous.

Let us consider graphs $G_{1}, G_{2}$ and a one-to-one partial graph mapping $F$ from $G_{1}$ to $G_{2}$. Now we state the propositions:

(i) $F^{-1} \mathbb{\mathbb { V }}=\left(F_{\mathbb{V}}\right)^{-1}$, and

(ii) $F^{-1} \mathbb{E}=\left(F_{\mathbb{E}}\right)^{-1}$.

(70) $\left(F^{-1}\right)^{-1}=F$.

(71) $F$ is total if and only if $F^{-1}$ is onto.

(72) $F$ is onto if and only if $F^{-1}$ is total.

(73) If $F$ is total and continuous, then $F^{-1}$ is continuous.

(74) If $F$ is total and directed-continuous, then $F^{-1}$ is directed-continuous.

(75) $F$ is isomorphism if and only if $F^{-1}$ is isomorphism.

(76) Let us consider w-graphs $G_{1}, G_{2}$, and a one-to-one partial graph mapping $F$ from $G_{1}$ to $G_{2}$. Then $F$ preserves weight if and only if $F^{-1}$ preserves weight. The theorem is a consequence of (2) and (70).

(77) Let us consider e-graphs $G_{1}, G_{2}$, and a one-to-one partial graph mapping $F$ from $G_{1}$ to $G_{2}$. Then $F$ preserves elabel if and only if $F^{-1}$ preserves elabel. The theorem is a consequence of (2) and (70).

(78) Let us consider v-graphs $G_{1}, G_{2}$, and a one-to-one partial graph mapping $F$ from $G_{1}$ to $G_{2}$. Then $F$ preserves vlabel if and only if $F^{-1}$ preserves vlabel. The theorem is a consequence of (2) and (70).

(79) Let us consider graphs $G_{1}, G_{2}$, and a one-to-one partial graph mapping $F$ from $G_{1}$ to $G_{2}$. Suppose $F$ is onto. Let us consider a vertex $v$ of $G_{2}$. Then $\left(F^{-1} \mathbb{V}\right)(v)$ is a vertex of $G_{1}$.

(80) Let us consider a graph $G$. Then $\left(\operatorname{id}_{G}\right)^{-1}=\operatorname{id}_{G}$.

(81) Let us consider graphs $G_{1}, G_{2}$, and a non empty, one-to-one partial graph mapping $F$ from $G_{1}$ to $G_{2}$. Then

(i) $\operatorname{dom} F=\operatorname{rng} F^{-1}$, and

(ii) $\operatorname{rng} F=\operatorname{dom}\left(F^{-1}\right)$.

The theorem is a consequence of (54). 
(82) Let us consider graphs $G_{1}, G_{2}$, a one-to-one partial graph mapping $F$ from $G_{1}$ to $G_{2}$, and a subgraph $H$ of $G_{1}$. Then $(F \nmid H)^{-1}=H 1 F^{-1}$.

(83) Let us consider graphs $G_{1}, G_{2}$, a one-to-one partial graph mapping $F$ from $G_{1}$ to $G_{2}$, and a subgraph $H$ of $G_{2}$. Then $(H \uparrow F)^{-1}=F^{-1}\lceil H$. The theorem is a consequence of (82) and (70).

(84) Let us consider graphs $G_{1}, G_{2}$, and a partial graph mapping $F$ from $G_{1}$ to $G_{2}$. Suppose $F$ is isomorphism. Then

(i) $G_{1} \cdot \operatorname{order}()=G_{2} \cdot \operatorname{order}()$, and

(ii) $G_{1} \cdot \operatorname{size}()=G_{2} \cdot \operatorname{size}()$.

The theorem is a consequence of (45) and (75).

(85) Let us consider finite graphs $G_{1}, G_{2}$, and a partial graph mapping $F$ from $G_{1}$ to $G_{2}$. Suppose $F$ is strong subgraph embedding. If there exists a partial graph mapping $F_{0}$ from $G_{1}$ to $G_{2}$ such that $F_{0}$ is isomorphism, then $F$ is isomorphism. The theorem is a consequence of (84) and (51).

(86) Let us consider graphs $G_{1}, G_{2}$, a partial graph mapping $F$ from $G_{1}$ to $G_{2}$, and subsets $X, Y$ of the vertices of $G_{1}$. Suppose $F$ is isomorphism. Then $\overline{\overline{G_{1} \text {.edgesBetween }(X, Y)}}=\overline{\overline{G_{2} \text {. edgesBetween }\left(\left(F_{\mathbb{V}}\right)^{\circ} X,\left(F_{\mathbb{V}}\right)^{\circ} Y\right)}}$. The theorem is a consequence of (46) and (75).

(87) Let us consider graphs $G_{1}, G_{2}$, a partial graph mapping $F$ from $G_{1}$ to $G_{2}$, and a subset $X$ of the vertices of $G_{1}$. Suppose $F$ is isomorphism. Then $\overline{\overline{G_{1} \text {.edgesBetween }(X)}}=\overline{\overline{G_{2} \text {.edgesBetween }\left(\left(F_{\mathbb{V}}\right)^{\circ} X\right)}}$. The theorem is a consequence of (47) and (75).

(88) Let us consider graphs $G_{1}, G_{2}$, a directed partial graph mapping $F$ from $G_{1}$ to $G_{2}$, and subsets $X, Y$ of the vertices of $G_{1}$. Suppose $F$ is isomorphism. Then $\overline{\overline{G_{1} \text {.edgesDBetween }(X, Y)}}=$ $\overline{G_{2} \text {. edgesDBetween }\left(\left(F_{\mathbb{V}}\right)^{\circ} X,\left(F_{\mathbb{V}}\right)^{\circ} Y\right)}$. The theorem is a consequence of (48) and (75).

Let us consider graphs $G_{1}, G_{2}$ and a partial graph mapping $F$ from $G_{1}$ to $G_{2}$. Now we state the propositions:

(89) Suppose $F$ is isomorphism. Then

(i) $G_{1}$ is trivial iff $G_{2}$ is trivial, and

(ii) $G_{1}$ is loopless iff $G_{2}$ is loopless, and

(iii) $G_{1}$ is edgeless iff $G_{2}$ is edgeless, and

(iv) $G_{1}$ is non-multi iff $G_{2}$ is non-multi, and

(v) $G_{1}$ is simple iff $G_{2}$ is simple, and

(vi) $G_{1}$ is finite iff $G_{2}$ is finite, and 
(vii) $G_{1}$ is complete iff $G_{2}$ is complete.

The theorem is a consequence of (75), (35), (49), and (52).

(90) Suppose $F$ is directed-continuous and isomorphism. Then

(i) $G_{1}$ is non-directed-multi iff $G_{2}$ is non-directed-multi, and

(ii) $G_{1}$ is directed-simple iff $G_{2}$ is directed-simple.

The theorem is a consequence of (74), (75), and (50).

(91) Let us consider graphs $G_{1}, G_{2}$, and a non empty, one-to-one partial graph mapping $F$ from $G_{1}$ to $G_{2}$. Then $\overline{\overline{\operatorname{dom} F \cdot \operatorname{loops}()}}=\overline{\overline{\operatorname{rng} F \cdot \operatorname{loops}()}}$. The theorem is a consequence of (81).

Let us consider graphs $G_{1}, G_{2}$ and a one-to-one partial graph mapping $F$ from $G_{1}$ to $G_{2}$. Now we state the propositions:

(92) If $F$ is total, then $\overline{\overline{G_{1} \cdot \operatorname{loops}()}} \subseteq \overline{\overline{G_{2} \cdot \operatorname{loops}()}}$. The theorem is a consequence of (55).

(93) If $F$ is onto, then $\overline{\overline{G_{2} \cdot \operatorname{loops}()}} \subseteq \overline{\overline{G_{1} \cdot \operatorname{loops}()}}$. The theorem is a consequence of (72) and (92).

(94) If $F$ is isomorphism, then $\overline{\overline{G_{1} \cdot \operatorname{loops}()}}=\overline{\overline{G_{2} \cdot \operatorname{loops}()}}$. The theorem is a consequence of (92) and (93).

(95) Let us consider a graph $G_{1}$, and a $G_{1}$-isomorphic graph $G_{2}$. Then $G_{1}$ is $G_{2}$-isomorphic. The theorem is a consequence of (75).

(96) Let us consider a graph $G_{1}$, and a $G_{1}$-directed-isomorphic graph $G_{2}$. Then $G_{1}$ is $G_{2}$-directed-isomorphic. The theorem is a consequence of (71) and $(72)$.

Let us consider a graph $G_{1}$, a $G_{1}$-isomorphic graph $G_{2}$, a $G_{2}$-isomorphic graph $G_{3}$, and an isomorphism $F$ between $G_{1}$ and $G_{2}$. Now we state the propositions:

(97) Suppose there exists a set $E$ such that $G_{3}$ is a graph given by reversing directions of the edges $E$ of $G_{1}$. Then $F^{-1}$ is an isomorphism between $G_{2}$ and $G_{3}$.

Proof: Reconsider $F_{2}=F^{-1}$ as a partial graph mapping from $G_{2}$ to $G_{3}$. $F_{2}$ is total. $F_{2}$ is onto.

(98) If $G_{1} \approx G_{3}$, then $F^{-1}$ is an isomorphism between $G_{2}$ and $G_{3}$. The theorem is a consequence of (97).

(99) Let us consider a graph $G_{1}$, a $G_{1}$-directed-isomorphic graph $G_{2}$, a $G_{2^{-}}$ directed-isomorphic graph $G_{3}$, and a directed isomorphism $F$ of $G_{1}$ and $G_{2}$. Suppose $G_{1} \approx G_{3}$. Then $F^{-1}$ is a directed isomorphism of $G_{2}$ and $G_{3}$. Proof: Reconsider $F_{2}=F^{-1}$ as a partial graph mapping from $G_{2}$ to $G_{3}$. $F_{2}$ is total. $F_{2}$ is onto. 
Let $G_{1}, G_{2}, G_{3}$ be graphs, $F_{1}$ be a partial graph mapping from $G_{1}$ to $G_{2}$, and $F_{2}$ be a partial graph mapping from $G_{2}$ to $G_{3}$. The functor $F_{2} \cdot F_{1}$ yielding a partial graph mapping from $G_{1}$ to $G_{3}$ is defined by the term

(Def. 34) $\left\langle\left(F_{2 \mathbb{V}}\right) \cdot\left(F_{1 \mathbb{V}}\right),\left(F_{2 \mathbb{E}}\right) \cdot\left(F_{1 \mathbb{E}}\right)\right\rangle$.

Let us consider graphs $G_{1}, G_{2}, G_{3}$, a partial graph mapping $F_{1}$ from $G_{1}$ to $G_{2}$, and a partial graph mapping $F_{2}$ from $G_{2}$ to $G_{3}$. Now we state the propositions:

(i) $F_{2} \cdot F_{1 \mathbb{V}}=\left(F_{2 \mathbb{V}}\right) \cdot\left(F_{1 \mathbb{V}}\right)$, and

(ii) $F_{2} \cdot F_{1 \mathbb{E}}=\left(F_{2 \mathbb{E}}\right) \cdot\left(F_{1 \mathbb{E}}\right)$.

(101) If $F_{2} \cdot F_{1}$ is onto, then $F_{2}$ is onto.

(102) If $F_{2} \cdot F_{1}$ is total, then $F_{1}$ is total.

Let $G_{1}, G_{2}, G_{3}$ be graphs, $F_{1}$ be a one-to-one partial graph mapping from $G_{1}$ to $G_{2}$, and $F_{2}$ be a one-to-one partial graph mapping from $G_{2}$ to $G_{3}$. Observe that $F_{2} \cdot F_{1}$ is one-to-one.

Let $F_{1}$ be a semi-continuous partial graph mapping from $G_{1}$ to $G_{2}$ and $F_{2}$ be a semi-continuous partial graph mapping from $G_{2}$ to $G_{3}$. Let us observe that $F_{2} \cdot F_{1}$ is semi-continuous.

Let $F_{1}$ be a continuous partial graph mapping from $G_{1}$ to $G_{2}$ and $F_{2}$ be a continuous partial graph mapping from $G_{2}$ to $G_{3}$. One can check that $F_{2} \cdot F_{1}$ is continuous.

Let $F_{1}$ be a directed partial graph mapping from $G_{1}$ to $G_{2}$ and $F_{2}$ be a directed partial graph mapping from $G_{2}$ to $G_{3}$. One can check that $F_{2} \cdot F_{1}$ is directed.

Let $F_{1}$ be a semi-directed-continuous partial graph mapping from $G_{1}$ to $G_{2}$ and $F_{2}$ be a semi-directed-continuous partial graph mapping from $G_{2}$ to $G_{3}$. Note that $F_{2} \cdot F_{1}$ is semi-directed-continuous.

Let $F_{1}$ be a directed-continuous partial graph mapping from $G_{1}$ to $G_{2}$ and $F_{2}$ be a directed-continuous partial graph mapping from $G_{2}$ to $G_{3}$. Observe that $F_{2} \cdot F_{1}$ is directed-continuous.

Let $F_{1}$ be an empty partial graph mapping from $G_{1}$ to $G_{2}$ and $F_{2}$ be a partial graph mapping from $G_{2}$ to $G_{3}$. Observe that $F_{2} \cdot F_{1}$ is empty.

Let $F_{1}$ be a partial graph mapping from $G_{1}$ to $G_{2}$ and $F_{2}$ be an empty partial graph mapping from $G_{2}$ to $G_{3}$. Let us observe that $F_{2} \cdot F_{1}$ is empty.

Let us consider graphs $G_{1}, G_{2}, G_{3}$, a partial graph mapping $F_{1}$ from $G_{1}$ to $G_{2}$, and a partial graph mapping $F_{2}$ from $G_{2}$ to $G_{3}$. Now we state the propositions:

(103) Suppose $F_{1}$ is total and $\operatorname{rng} F_{1 \mathbb{V}} \subseteq \operatorname{dom}\left(F_{2 \mathbb{V}}\right)$ and $\operatorname{rng} F_{1 \mathbb{E}} \subseteq \operatorname{dom}\left(F_{2 \mathbb{E}}\right)$. Then $F_{2} \cdot F_{1}$ is total. 
(104) If $F_{1}$ is total and $F_{2}$ is total, then $F_{2} \cdot F_{1}$ is total. The theorem is a consequence of (103).

(105) Suppose $F_{2}$ is onto and $\operatorname{dom}\left(F_{2 \mathbb{V}}\right) \subseteq \operatorname{rng} F_{1 \mathbb{V}}$ and $\operatorname{dom}\left(F_{2 \mathbb{E}}\right) \subseteq \operatorname{rng} F_{1 \mathbb{E}}$. Then $F_{2} \cdot F_{1}$ is onto.

(106) If $F_{1}$ is onto and $F_{2}$ is onto, then $F_{2} \cdot F_{1}$ is onto. The theorem is a consequence of (105).

(107) If $F_{1}$ is weak subgraph embedding and $F_{2}$ is weak subgraph embedding, then $F_{2} \cdot F_{1}$ is weak subgraph embedding.

(108) If $F_{1}$ is strong subgraph embedding and $F_{2}$ is strong subgraph embedding, then $F_{2} \cdot F_{1}$ is strong subgraph embedding.

(109) If $F_{1}$ is isomorphism and $F_{2}$ is isomorphism, then $F_{2} \cdot F_{1}$ is isomorphism.

(110) If $F_{1}$ is directed-isomorphism and $F_{2}$ is directed-isomorphism, then $F_{2} \cdot F_{1}$ is directed-isomorphism. The theorem is a consequence of (109).

(111) Let us consider w-graphs $G_{1}, G_{2}, G_{3}$, a partial graph mapping $F_{1}$ from $G_{1}$ to $G_{2}$, and a partial graph mapping $F_{2}$ from $G_{2}$ to $G_{3}$. Suppose $F_{1}$ preserves weight and $F_{2}$ preserves weight. Then $F_{2} \cdot F_{1}$ preserves weight. The theorem is a consequence of (1).

(112) Let us consider e-graphs $G_{1}, G_{2}, G_{3}$, a partial graph mapping $F_{1}$ from $G_{1}$ to $G_{2}$, and a partial graph mapping $F_{2}$ from $G_{2}$ to $G_{3}$. Suppose $F_{1}$ preserves elabel and $F_{2}$ preserves elabel. Then $F_{2} \cdot F_{1}$ preserves elabel. The theorem is a consequence of (1).

(113) Let us consider v-graphs $G_{1}, G_{2}, G_{3}$, a partial graph mapping $F_{1}$ from $G_{1}$ to $G_{2}$, and a partial graph mapping $F_{2}$ from $G_{2}$ to $G_{3}$. Suppose $F_{1}$ preserves vlabel and $F_{2}$ preserves vlabel. Then $F_{2} \cdot F_{1}$ preserves vlabel. The theorem is a consequence of (1).

(114) Let us consider graphs $G_{1}, G_{2}, G_{3}, G_{4}$, a partial graph mapping $F_{1}$ from $G_{1}$ to $G_{2}$, a partial graph mapping $F_{2}$ from $G_{2}$ to $G_{3}$, and a partial graph mapping $F_{3}$ from $G_{3}$ to $G_{4}$. Then $F_{3} \cdot\left(F_{2} \cdot F_{1}\right)=\left(F_{3} \cdot F_{2}\right) \cdot F_{1}$.

(115) Let us consider graphs $G_{1}, G_{2}$, and a one-to-one partial graph mapping $F$ from $G_{1}$ to $G_{2}$. Suppose $F$ is isomorphism. Then

(i) $F \cdot\left(F^{-1}\right)=\operatorname{id}_{G_{2}}$, and

(ii) $F^{-1} \cdot F=\operatorname{id}_{G_{1}}$.

(116) Let us consider graphs $G_{1}, G_{2}$, and a partial graph mapping $F$ from $G_{1}$ to $G_{2}$. Then

(i) $F \cdot\left(\operatorname{id}_{G_{1}}\right)=F$, and

(ii) $\operatorname{id}_{G_{2}} \cdot F=F$. 
(117) Let us consider graphs $G_{1}, G_{2}, G_{3}$, a partial graph mapping $F_{1}$ from $G_{1}$ to $G_{2}$, a partial graph mapping $F_{2}$ from $G_{2}$ to $G_{3}$, and a subgraph $H$ of $G_{1}$. Then $F_{2} \cdot\left(F_{1}\lceil H)=\left(F_{2} \cdot F_{1}\right)\lceil H\right.$.

(118) Let us consider graphs $G_{1}, G_{2}, G_{3}$, a partial graph mapping $F_{1}$ from $G_{1}$ to $G_{2}$, a partial graph mapping $F_{2}$ from $G_{2}$ to $G_{3}$, and a subgraph $H$ of $G_{3}$. Then $\left(H 1 F_{2}\right) \cdot F_{1}=H 1\left(F_{2} \cdot F_{1}\right)$.

Let $G_{1}$ be a graph and $G_{2}$ be a $G_{1}$-isomorphic graph. Let us note that every graph which is $G_{2}$-isomorphic is also $G_{1}$-isomorphic.

Let $G_{2}$ be a $G_{1}$-directed-isomorphic graph. Note that every graph which is $G_{2}$-directed-isomorphic is also $G_{1}$-directed-isomorphic.

\section{Walks Induced by Graph Mappings}

Let $G_{1}, G_{2}$ be graphs, $F$ be a partial graph mapping from $G_{1}$ to $G_{2}$, and $W_{1}$ be a walk of $G_{1}$. We say that $W_{1}$ is $F$-defined if and only if

(Def. 35) $W_{1} \cdot \operatorname{vertices}() \subseteq \operatorname{dom}\left(F_{\mathbb{V}}\right)$ and $W_{1} \cdot \operatorname{edges}() \subseteq \operatorname{dom}\left(F_{\mathbb{E}}\right)$.

Let $W_{2}$ be a walk of $G_{2}$. We say that $W_{2}$ is $F$-valued if and only if

(Def. 36) $\quad W_{2}$.vertices ()$\subseteq \operatorname{rng} F_{\mathbb{V}}$ and $W_{2}$.edges ()$\subseteq \operatorname{rng} F_{\mathbb{E}}$.

Let $F$ be a non empty partial graph mapping from $G_{1}$ to $G_{2}$. Observe that there exists a walk of $G_{1}$ which is $F$-defined and trivial and there exists a walk of $G_{2}$ which is $F$-valued and trivial.

Let us consider graphs $G_{1}, G_{2}$ and an empty partial graph mapping $F$ from $G_{1}$ to $G_{2}$. Now we state the propositions:

(119) Every walk of $G_{1}$ is not $F$-defined.

(120) Every walk of $G_{2}$ is not $F$-valued.

(121) Let us consider graphs $G_{1}, G_{2}$, a partial graph mapping $F$ from $G_{1}$ to $G_{2}$, and a walk $W_{1}$ of $G_{1}$. If $F$ is total, then $W_{1}$ is $F$-defined.

(122) Let us consider graphs $G_{1}, G_{2}$, a partial graph mapping $F$ from $G_{1}$ to $G_{2}$, and a walk $W_{2}$ of $G_{2}$. If $F$ is onto, then $W_{2}$ is $F$-valued.

Let $G_{1}, G_{2}$ be graphs and $F$ be a one-to-one partial graph mapping from $G_{1}$ to $G_{2}$. Observe that every walk of $G_{1}$ which is $F$-defined is also $\left(F^{-1}\right)$-valued and every walk of $G_{2}$ which is $F$-valued is also $\left(F^{-1}\right)$-defined.

Let $F$ be a non empty partial graph mapping from $G_{1}$ to $G_{2}$ and $W_{1}$ be an $F$-defined walk of $G_{1}$. The functor $F^{\circ} W_{1}$ yielding a walk of $G_{2}$ is defined by

$\left(\right.$ Def. 37) $\left(F_{\mathbb{V}}\right) \cdot\left(W_{1} \cdot \operatorname{vertexSeq}()\right)=i t \cdot \operatorname{vertexSeq}()$ and $\left(F_{\mathbb{E}}\right) \cdot\left(W_{1} \cdot \operatorname{edgeSeq}()\right)=$ it.edgeSeq().

Note that $F^{\circ} W_{1}$ is $F$-valued. 
Let us observe that the functor $F^{\circ} W_{1}$ yields an $F$-valued walk of $G_{2}$. Let $F$ be a non empty, one-to-one partial graph mapping from $G_{1}$ to $G_{2}$ and $W_{2}$ be an $F$-valued walk of $G_{2}$. The functor $F^{-1}\left(W_{2}\right)$ yielding an $F$-defined walk of $G_{1}$ is defined by the term

(Def. 38) $\left(F^{-1}\right)^{\circ} W_{2}$.

Let us observe that the functor $F^{-1}\left(W_{2}\right)$ is defined by

$\left(\right.$ Def. 39) $\left(F_{\mathbb{V}}\right) \cdot(i t$.vertexSeq ()$)=W_{2}$.vertexSeq () and $\left(F_{\mathbb{E}}\right) \cdot(i t$.edgeSeq ()$)=$ $W_{2} \cdot \operatorname{edgeSeq}()$.

Now we state the propositions:

(123) Let us consider graphs $G_{1}, G_{2}$, a non empty, one-to-one partial graph mapping $F$ from $G_{1}$ to $G_{2}$, and an $F$-defined walk $W_{1}$ of $G_{1}$. Then $F^{-1}\left(F^{\circ} W_{1}\right)=W_{1}$.

(124) Let us consider graphs $G_{1}, G_{2}$, a non empty, one-to-one partial graph mapping $F$ from $G_{1}$ to $G_{2}$, and an $F$-valued walk $W_{2}$ of $G_{2}$.

Then $F^{\circ}\left(F^{-1}\left(W_{2}\right)\right)=W_{2}$.

(125) Let us consider graphs $G_{1}, G_{2}$, a non empty partial graph mapping $F$ from $G_{1}$ to $G_{2}$, and an $F$-defined walk $W_{1}$ of $G_{1}$. Then

(i) $W_{1} \cdot \operatorname{length}()=\left(F^{\circ} W_{1}\right) \cdot \operatorname{length}()$, and

(ii) len $W_{1}=\operatorname{len}\left(F^{\circ} W_{1}\right)$.

(126) Let us consider graphs $G_{1}, G_{2}$, a non empty, one-to-one partial graph mapping $F$ from $G_{1}$ to $G_{2}$, and an $F$-valued walk $W_{2}$ of $G_{2}$. Then

(i) $W_{2} \cdot \operatorname{length}()=\left(F^{-1}\left(W_{2}\right)\right) \cdot \operatorname{length}()$, and

(ii) len $W_{2}=\operatorname{len}\left(F^{-1}\left(W_{2}\right)\right)$.

(127) Let us consider graphs $G_{1}, G_{2}$, a non empty partial graph mapping $F$ from $G_{1}$ to $G_{2}$, and an $F$-defined walk $W_{1}$ of $G_{1}$. Then

(i) $\left(F_{\mathbb{V}}\right)\left(W_{1} \cdot \operatorname{first}()\right)=\left(F^{\circ} W_{1}\right)$.first () , and

(ii) $\left(F_{\mathbb{V}}\right)\left(W_{1} \cdot \operatorname{last}()\right)=\left(F^{\circ} W_{1}\right) \cdot \operatorname{last}()$.

(128) Let us consider graphs $G_{1}, G_{2}$, a non empty, one-to-one partial graph mapping $F$ from $G_{1}$ to $G_{2}$, and an $F$-valued walk $W_{2}$ of $G_{2}$. Then

(i) $\left(\left(F_{\mathbb{V}}\right)^{-1}\right)\left(W_{2}\right.$.first ()$)=\left(F^{-1}\left(W_{2}\right)\right)$.first () , and

(ii) $\left(\left(F_{\mathbb{V}}\right)^{-1}\right)\left(W_{2} \cdot \operatorname{last}()\right)=\left(F^{-1}\left(W_{2}\right)\right) \cdot \operatorname{last}()$.

(129) Let us consider graphs $G_{1}, G_{2}$, a non empty partial graph mapping $F$ from $G_{1}$ to $G_{2}$, an $F$-defined walk $W_{1}$ of $G_{1}$, and an odd element $n$ of $\mathbb{N}$. If $n \leqslant$ len $W_{1}$, then $\left(F_{\mathbb{V}}\right)\left(W_{1}(n)\right)=\left(F^{\circ} W_{1}\right)(n)$. The theorem is a consequence of (125). 
(130) Let us consider graphs $G_{1}, G_{2}$, a non empty partial graph mapping $F$ from $G_{1}$ to $G_{2}$, an $F$-defined walk $W_{1}$ of $G_{1}$, and an even element $n$ of $\mathbb{N}$. Suppose $1 \leqslant n \leqslant$ len $W_{1}$. Then $\left(F_{\mathbb{E}}\right)\left(W_{1}(n)\right)=\left(F^{\circ} W_{1}\right)(n)$. The theorem is a consequence of (125).

Let us consider graphs $G_{1}, G_{2}$, a non empty partial graph mapping $F$ from $G_{1}$ to $G_{2}$, an $F$-defined walk $W_{1}$ of $G_{1}$, and objects $v, w$. Now we state the propositions:

(131) If $W_{1}$ is walk from $v$ to $w$, then $v, w \in \operatorname{dom}\left(F_{\mathbb{V}}\right)$.

(132) If $W_{1}$ is walk from $v$ to $w$, then $F^{\circ} W_{1}$ is walk from $\left(F_{\mathbb{V}}\right)(v)$ to $\left(F_{\mathbb{V}}\right)(w)$. The theorem is a consequence of (129) and (125).

(133) Let us consider graphs $G_{1}, G_{2}$, a non empty, one-to-one partial graph mapping $F$ from $G_{1}$ to $G_{2}$, an $F$-defined walk $W_{1}$ of $G_{1}$, and objects $v$, $w$. Then $W_{1}$ is walk from $v$ to $w$ if and only if $v, w \in \operatorname{dom}\left(F_{\mathbb{V}}\right)$ and $F^{\circ} W_{1}$ is walk from $\left(F_{\mathbb{V}}\right)(v)$ to $\left(F_{\mathbb{V}}\right)(w)$. The theorem is a consequence of $(131)$, (132), and (123).

(134) Let us consider graphs $G_{1}, G_{2}$, a non empty, one-to-one partial graph mapping $F$ from $G_{1}$ to $G_{2}$, and an $F$-defined walk $W_{1}$ of $G_{1}$. Suppose $\left(F_{\mathbb{V}}\right)\left(W_{1}\right.$.first ()$)=\left(F_{\mathbb{V}}\right)\left(W_{1}\right.$.last ()$)$. Then $W_{1}$.first ()$=W_{1}$.last () . The theorem is a consequence of (4).

Let us consider graphs $G_{1}, G_{2}$, a non empty partial graph mapping $F$ from $G_{1}$ to $G_{2}$, and an $F$-defined walk $W_{1}$ of $G_{1}$. Now we state the propositions:

(135) $\quad\left(F^{\circ} W_{1}\right) \cdot \operatorname{vertices}()=\left(F_{\mathbb{V}}\right)^{\circ}\left(W_{1} \cdot \operatorname{vertices}()\right)$.

Proof: For every object $y, y \in \operatorname{rng}\left(F_{\mathbb{V}}\right) \cdot\left(W_{1}\right.$.vertexSeq ()$)$ iff $y \in$ $\left(F_{\mathbb{V}}\right)^{\circ}\left(W_{1}\right.$.vertices ()$)$.

(136) $\quad\left(F^{\circ} W_{1}\right) \cdot$ edges ()$=\left(F_{\mathbb{E}}\right)^{\circ}\left(W_{1} \cdot\right.$ edges ()$)$.

Proof: For every object $y, y \in \operatorname{rng}\left(F_{\mathbb{E}}\right) \cdot\left(W_{1}\right.$.edgeSeq ()$)$ iff $y \in$ $\left(F_{\mathbb{E}}\right)^{\circ}\left(W_{1}\right.$.edges ()$)$.

(137) (i) if $W_{1}$ is trivial, then $F^{\circ} W_{1}$ is trivial, and

(ii) if $W_{1}$ is closed, then $F^{\circ} W_{1}$ is closed, and

(iii) if $F^{\circ} W_{1}$ is trail-like, then $W_{1}$ is trail-like, and

(iv) if $F^{\circ} W_{1}$ is path-like, then $W_{1}$ is path-like.

Proof: If $F^{\circ} W_{1}$ is trail-like, then $W_{1}$ is trail-like. For every odd elements $m, n$ of $\mathbb{N}$ such that $m<n \leqslant$ len $W_{1}$ holds if $W_{1}(m)=W_{1}(n)$, then $m=1$ and $n=$ len $W_{1}$.

(138) Let us consider graphs $G_{1}, G_{2}$, a non empty, one-to-one partial graph mapping $F$ from $G_{1}$ to $G_{2}$, and an $F$-defined walk $W_{1}$ of $G_{1}$. Then

(i) $W_{1}$ is trivial iff $F^{\circ} W_{1}$ is trivial, and 
(ii) $W_{1}$ is closed iff $F^{\circ} W_{1}$ is closed, and

(iii) $W_{1}$ is trail-like iff $F^{\circ} W_{1}$ is trail-like, and

(iv) $W_{1}$ is path-like iff $F^{\circ} W_{1}$ is path-like, and

(v) $W_{1}$ is circuit-like iff $F^{\circ} W_{1}$ is circuit-like, and

(vi) $W_{1}$ is cycle-like iff $F^{\circ} W_{1}$ is cycle-like.

The theorem is a consequence of (123) and (137).

Let us consider graphs $G_{1}, G_{2}$ and a partial graph mapping $F$ from $G_{1}$ to $G_{2}$. Now we state the propositions:

(139) If $F$ is strong subgraph embedding, then if $G_{2}$ is acyclic, then $G_{1}$ is acyclic. The theorem is a consequence of (121) and (138).

(140) Suppose $F$ is isomorphism. Then

(i) $G_{1}$ is acyclic iff $G_{2}$ is acyclic, and

(ii) $G_{1}$ is chordal iff $G_{2}$ is chordal, and

(iii) $G_{1}$ is connected iff $G_{2}$ is connected.

Proof: $F^{-1}$ is isomorphism and semi-continuous. For every vertices $u, v$ of $G_{1}$, there exists a walk $W_{1}$ of $G_{1}$ such that $W_{1}$ is walk from $u$ to $v$.

\section{Graph Mappings and Graph Modes}

Let us consider graphs $G_{1}, G_{2}$, sets $E_{1}, E_{2}$, a graph $G_{3}$ given by reversing directions of the edges $E_{1}$ of $G_{1}$, a graph $G_{4}$ given by reversing directions of the edges $E_{2}$ of $G_{2}$, and a partial graph mapping $F_{0}$ from $G_{1}$ to $G_{2}$. Now we state the propositions:

(141) There exists a partial graph mapping $F$ from $G_{3}$ to $G_{4}$ such that

(i) $F=F_{0}$, and

(ii) if $F_{0}$ is not empty, then $F$ is not empty, and

(iii) if $F_{0}$ is total, then $F$ is total, and

(iv) if $F_{0}$ is onto, then $F$ is onto, and

(v) if $F_{0}$ is one-to-one, then $F$ is one-to-one, and

(vi) if $F_{0}$ is semi-continuous, then $F$ is semi-continuous, and

(vii) if $F_{0}$ is continuous, then $F$ is continuous.

Proof: Reconsider $F=F_{0}$ as a partial graph mapping from $G_{3}$ to $G_{4}$. If $F_{0}$ is semi-continuous, then $F$ is semi-continuous. If $F_{0}$ is continuous, then $F$ is continuous by [13, (9)]. 
(142) There exists a partial graph mapping $F$ from $G_{3}$ to $G_{4}$ such that

(i) $F=F_{0}$, and

(ii) if $F_{0}$ is weak subgraph embedding, then $F$ is weak subgraph embedding, and

(iii) if $F_{0}$ is strong subgraph embedding, then $F$ is strong subgraph embedding, and

(iv) if $F_{0}$ is isomorphism, then $F$ is isomorphism.

The theorem is a consequence of (141).

(143) Let us consider a graph $G_{1}$, a $G_{1}$-isomorphic graph $G_{2}$, sets $E_{1}, E_{2}$, and a graph $G_{3}$ given by reversing directions of the edges $E_{1}$ of $G_{1}$. Then every graph given by reversing directions of the edges $E_{2}$ of $G_{2}$ is $G_{3}$-isomorphic.

The theorem is a consequence of (142).

Let us consider graphs $G_{3}, G_{4}$, sets $V_{1}, V_{2}$, a supergraph $G_{1}$ of $G_{3}$ extended by the vertices from $V_{1}$, a supergraph $G_{2}$ of $G_{4}$ extended by the vertices from $V_{2}$, a partial graph mapping $F_{0}$ from $G_{3}$ to $G_{4}$, and a one-to-one function $f$. Now we state the propositions:

(144) Suppose $\operatorname{dom} f=V_{1} \backslash$ (the vertices of $\left.G_{3}\right)$ and $\operatorname{rng} f=V_{2} \backslash$ (the vertices of $G_{4}$ ). Then there exists a partial graph mapping $F$ from $G_{1}$ to $G_{2}$ such that

(i) $F=\left\langle F_{0 \mathbb{V}}+\cdot f, F_{0 \mathbb{E}}\right\rangle$, and

(ii) if $F_{0}$ is not empty, then $F$ is not empty, and

(iii) if $F_{0}$ is total, then $F$ is total, and

(iv) if $F_{0}$ is onto, then $F$ is onto, and

(v) if $F_{0}$ is one-to-one, then $F$ is one-to-one, and

(vi) if $F_{0}$ is directed, then $F$ is directed, and

(vii) if $F_{0}$ is semi-continuous, then $F$ is semi-continuous, and

(viii) if $F_{0}$ is continuous, then $F$ is continuous, and

(ix) if $F_{0}$ is semi-directed-continuous, then $F$ is semi-directed-continuous, and

(x) if $F_{0}$ is directed-continuous, then $F$ is directed-continuous.

Proof: Set $h=F_{0 \mathbb{V}}+\cdot f$. Reconsider $g=F_{0 \mathbb{E}}$ as a partial function from the edges of $G_{1}$ to the edges of $G_{2}$. Reconsider $F=\langle h, g\rangle$ as a partial graph mapping from $G_{1}$ to $G_{2}$. If $F_{0}$ is total, then $F$ is total. If $F_{0}$ is onto, then $F$ is onto. If $F_{0}$ is directed, then $F$ is directed. If $F_{0}$ is semi-continuous, then $F$ is semi-continuous. If $F_{0}$ is continuous, then $F$ is continuous. If $F_{0}$ is semi-directed-continuous, then $F$ is semi-directed-continuous. If $F_{0}$ is directed-continuous, then $F$ is directed-continuous. 
(145) Suppose $\operatorname{dom} f=V_{1} \backslash$ (the vertices of $G_{3}$ ) and $\operatorname{rng} f=V_{2} \backslash$ (the vertices of $G_{4}$ ). Then there exists a partial graph mapping $F$ from $G_{1}$ to $G_{2}$ such that

(i) $F=\left\langle F_{0 \mathbb{V}}+\cdot f, F_{0 \mathbb{E}}\right\rangle$, and

(ii) if $F_{0}$ is weak subgraph embedding, then $F$ is weak subgraph embedding, and

(iii) if $F_{0}$ is strong subgraph embedding, then $F$ is strong subgraph embedding, and

(iv) if $F_{0}$ is isomorphism, then $F$ is isomorphism, and

(v) if $F_{0}$ is directed-isomorphism, then $F$ is directed-isomorphism.

The theorem is a consequence of (144).

(146) Let us consider a graph $G_{3}$, a $G_{3}$-isomorphic graph $G_{4}$, sets $V_{1}, V_{2}$, a supergraph $G_{1}$ of $G_{3}$ extended by the vertices from $V_{1}$, and a supergraph $G_{2}$ of $G_{4}$ extended by the vertices from $V_{2}$. Suppose $\overline{\overline{V_{1} \backslash \alpha}}=\overline{\overline{V_{2} \backslash \beta}}$. Then $G_{2}$ is $G_{1}$-isomorphic, where $\alpha$ is the vertices of $G_{3}$ and $\beta$ is the vertices of $G_{4}$. The theorem is a consequence of (145).

(147) Let us consider a graph $G_{3}$, a $G_{3}$-directed-isomorphic graph $G_{4}$, sets $V_{1}, V_{2}$, a supergraph $G_{1}$ of $G_{3}$ extended by the vertices from $V_{1}$, and a supergraph $G_{2}$ of $G_{4}$ extended by the vertices from $V_{2}$. Suppose $\overline{\overline{V_{1} \backslash \alpha}}=$ $\overline{\overline{V_{2} \backslash \beta}}$. Then $G_{2}$ is $G_{1}$-directed-isomorphic, where $\alpha$ is the vertices of $G_{3}$ and $\beta$ is the vertices of $G_{4}$. The theorem is a consequence of (145).

Let us consider graphs $G_{3}, G_{4}$, objects $v_{1}, v_{2}$, a supergraph $G_{1}$ of $G_{3}$ extended by $v_{1}$, a supergraph $G_{2}$ of $G_{4}$ extended by $v_{2}$, and a partial graph mapping $F_{0}$ from $G_{3}$ to $G_{4}$. Now we state the propositions:

(148) Suppose $v_{1} \notin$ the vertices of $G_{3}$ and $v_{2} \notin$ the vertices of $G_{4}$. Then there exists a partial graph mapping $F$ from $G_{1}$ to $G_{2}$ such that

(i) $F=\left\langle F_{0 \mathbb{V}}+\cdot\left(v_{1} \longmapsto v_{2}\right), F_{0 \mathbb{E}}\right\rangle$, and

(ii) if $F_{0}$ is total, then $F$ is total, and

(iii) if $F_{0}$ is onto, then $F$ is onto, and

(iv) if $F_{0}$ is one-to-one, then $F$ is one-to-one, and

(v) if $F_{0}$ is directed, then $F$ is directed, and

(vi) if $F_{0}$ is semi-continuous, then $F$ is semi-continuous, and

(vii) if $F_{0}$ is continuous, then $F$ is continuous, and

(viii) if $F_{0}$ is semi-directed-continuous, then $F$ is semi-directed-continuous, and 
(ix) if $F_{0}$ is directed-continuous, then $F$ is directed-continuous.

The theorem is a consequence of (144).

(149) Suppose $v_{1} \notin$ the vertices of $G_{3}$ and $v_{2} \notin$ the vertices of $G_{4}$. Then there exists a partial graph mapping $F$ from $G_{1}$ to $G_{2}$ such that

(i) $F=\left\langle F_{0 \mathbb{V}}+\cdot\left(v_{1} \longmapsto v_{2}\right), F_{0 \mathbb{E}}\right\rangle$, and

(ii) if $F_{0}$ is weak subgraph embedding, then $F$ is weak subgraph embedding, and

(iii) if $F_{0}$ is strong subgraph embedding, then $F$ is strong subgraph embedding, and

(iv) if $F_{0}$ is isomorphism, then $F$ is isomorphism, and

(v) if $F_{0}$ is directed-isomorphism, then $F$ is directed-isomorphism.

The theorem is a consequence of (148).

(150) Let us consider a graph $G_{3}$, a $G_{3}$-isomorphic graph $G_{4}$, objects $v_{1}, v_{2}$, a supergraph $G_{1}$ of $G_{3}$ extended by $v_{1}$, and a supergraph $G_{2}$ of $G_{4}$ extended by $v_{2}$. Suppose $v_{1} \in$ the vertices of $G_{3}$ iff $v_{2} \in$ the vertices of $G_{4}$. Then $G_{2}$ is $G_{1}$-isomorphic. The theorem is a consequence of (146).

(151) Let us consider a graph $G_{3}$, a $G_{3}$-directed-isomorphic graph $G_{4}$, objects $v_{1}, v_{2}$, a supergraph $G_{1}$ of $G_{3}$ extended by $v_{1}$, and a supergraph $G_{2}$ of $G_{4}$ extended by $v_{2}$. Suppose $v_{1} \in$ the vertices of $G_{3}$ iff $v_{2} \in$ the vertices of $G_{4}$. Then $G_{2}$ is $G_{1}$-directed-isomorphic. The theorem is a consequence of (147).

Let us consider graphs $G_{3}, G_{4}$, vertices $v_{1}, v_{3}$ of $G_{3}$, vertices $v_{2}, v_{4}$ of $G_{4}$, objects $e_{1}, e_{2}$, a supergraph $G_{1}$ of $G_{3}$ extended by $e_{1}$ between vertices $v_{1}$ and $v_{3}$, a supergraph $G_{2}$ of $G_{4}$ extended by $e_{2}$ between vertices $v_{2}$ and $v_{4}$, and a partial graph mapping $F_{0}$ from $G_{3}$ to $G_{4}$. Now we state the propositions:

(152) Suppose $e_{1} \notin$ the edges of $G_{3}$ and $e_{2} \notin$ the edges of $G_{4}$ and $v_{1}, v_{3} \in$ $\operatorname{dom}\left(F_{0 \mathbb{V}}\right)$ and $\left(\left(F_{0 \mathbb{V}}\right)\left(v_{1}\right)=v_{2}\right.$ and $\left(F_{0 \mathbb{V}}\right)\left(v_{3}\right)=v_{4}$ or $\left(F_{0 \mathbb{V}}\right)\left(v_{1}\right)=v_{4}$ and $\left.\left(F_{0 \mathbb{V}}\right)\left(v_{3}\right)=v_{2}\right)$. Then there exists a partial graph mapping $F$ from $G_{1}$ to $G_{2}$ such that

(i) $F=\left\langle F_{0 \mathbb{V}}, F_{0 \mathbb{E}}+\cdot\left(e_{1} \longmapsto e_{2}\right)\right\rangle$, and

(ii) if $F_{0}$ is total, then $F$ is total, and

(iii) if $F_{0}$ is onto, then $F$ is onto, and

(iv) if $F_{0}$ is one-to-one, then $F$ is one-to-one.

The theorem is a consequence of (5), (4), and (8).

(153) Suppose $e_{1} \notin$ the edges of $G_{3}$ and $e_{2} \notin$ the edges of $G_{4}$ and $v_{1}, v_{3} \in$ $\operatorname{dom}\left(F_{0 \mathbb{V}}\right)$ and $\left(\left(F_{0 \mathbb{V}}\right)\left(v_{1}\right)=v_{2}\right.$ and $\left(F_{0 \mathbb{V}}\right)\left(v_{3}\right)=v_{4}$ or $\left(F_{0 \mathbb{V}}\right)\left(v_{1}\right)=v_{4}$ and 
$\left.\left(F_{0 \mathbb{V}}\right)\left(v_{3}\right)=v_{2}\right)$. Then there exists a partial graph mapping $F$ from $G_{1}$ to $G_{2}$ such that

(i) $F=\left\langle F_{0 \mathbb{V}}, F_{0 \mathbb{E}}+\cdot\left(e_{1} \longmapsto e_{2}\right)\right\rangle$, and

(ii) if $F_{0}$ is weak subgraph embedding, then $F$ is weak subgraph embedding, and

(iii) if $F_{0}$ is isomorphism, then $F$ is isomorphism.

The theorem is a consequence of (152).

(154) Suppose $e_{1} \notin$ the edges of $G_{3}$ and $e_{2} \notin$ the edges of $G_{4}$ and $v_{1}, v_{3} \in$ $\operatorname{dom}\left(F_{0 \mathbb{V}}\right)$ and $\left(F_{0 \mathbb{V}}\right)\left(v_{1}\right)=v_{2}$ and $\left(F_{0 \mathbb{V}}\right)\left(v_{3}\right)=v_{4}$. Then there exists a partial graph mapping $F$ from $G_{1}$ to $G_{2}$ such that

(i) $F=\left\langle F_{0 \mathbb{V}}, F_{0 \mathbb{E}}+\cdot\left(e_{1} \longmapsto e_{2}\right)\right\rangle$, and

(ii) if $F_{0}$ is directed, then $F$ is directed, and

(iii) if $F_{0}$ is directed-isomorphism, then $F$ is directed-isomorphism.

Proof: Consider $F$ being a partial graph mapping from $G_{1}$ to $G_{2}$ such that $F=\left\langle F_{0 \mathbb{V}}, F_{0 \mathbb{E}}+\cdot\left(e_{1} \longmapsto e_{2}\right)\right\rangle$ and if $F_{0}$ is total, then $F$ is total and if $F_{0}$ is onto, then $F$ is onto and if $F_{0}$ is one-to-one, then $F$ is one-to-one. If $F_{0}$ is directed, then $F$ is directed by [15, (16)], [12, (71),(70),(106)].

Let us consider graphs $G_{3}, G_{4}$, a vertex $v_{3}$ of $G_{3}$, a vertex $v_{4}$ of $G_{4}$, objects $e_{1}, e_{2}, v_{1}, v_{2}$, a supergraph $G_{1}$ of $G_{3}$ extended by $v_{1}, v_{3}$ and $e_{1}$ between them, a supergraph $G_{2}$ of $G_{4}$ extended by $v_{2}, v_{4}$ and $e_{2}$ between them, and a partial graph mapping $F_{0}$ from $G_{3}$ to $G_{4}$. Now we state the propositions:

(155) Suppose $e_{1} \notin$ the edges of $G_{3}$ and $e_{2} \notin$ the edges of $G_{4}$ and $v_{1} \notin$ the vertices of $G_{3}$ and $v_{2} \notin$ the vertices of $G_{4}$ and $v_{3} \in \operatorname{dom}\left(F_{0 \mathbb{V}}\right)$ and $\left(F_{0 \mathbb{V}}\right)\left(v_{3}\right)=v_{4}$. Then there exists a partial graph mapping $F$ from $G_{1}$ to $G_{2}$ such that

(i) $F=\left\langle F_{0 \mathbb{V}}+\cdot\left(v_{1} \longmapsto v_{2}\right), F_{0 \mathbb{E}}+\cdot\left(e_{1} \longmapsto e_{2}\right)\right\rangle$, and

(ii) if $F_{0}$ is total, then $F$ is total, and

(iii) if $F_{0}$ is onto, then $F$ is onto, and

(iv) if $F_{0}$ is one-to-one, then $F$ is one-to-one, and

(v) if $F_{0}$ is directed, then $F$ is directed.

Proof: Consider $G_{5}$ being a supergraph of $G_{3}$ extended by $v_{1}$ such that $G_{1}$ is a supergraph of $G_{5}$ extended by $e_{1}$ between vertices $v_{1}$ and $v_{3}$. Consider $G_{6}$ being a supergraph of $G_{4}$ extended by $v_{2}$ such that $G_{2}$ is a supergraph of $G_{6}$ extended by $e_{2}$ between vertices $v_{2}$ and $v_{4}$.

Consider $F_{1}$ being a partial graph mapping from $G_{5}$ to $G_{6}$ such that $F_{1}=\left\langle F_{0 \mathbb{V}}+\cdot\left(v_{1} \longmapsto v_{2}\right), F_{0 \mathbb{E}}\right\rangle$ and if $F_{0}$ is total, then $F_{1}$ is total and if $F_{0}$ 
is onto, then $F_{1}$ is onto and if $F_{0}$ is one-to-one, then $F_{1}$ is one-to-one and if $F_{0}$ is directed, then $F_{1}$ is directed and if $F_{0}$ is semi-continuous, then $F_{1}$ is semi-continuous and if $F_{0}$ is continuous, then $F_{1}$ is continuous and if $F_{0}$ is semi-directed-continuous, then $F_{1}$ is semi-directed-continuous and if $F_{0}$ is directed-continuous, then $F_{1}$ is directed-continuous. $v_{1}, v_{3} \in \operatorname{dom}\left(F_{1 \mathbb{V}}\right)$ and $\left(F_{1 \mathbb{V}}\right)\left(v_{1}\right)=v_{2}$ and $\left(F_{1 \mathbb{V}}\right)\left(v_{3}\right)=v_{4}$.

Consider $F_{2}$ being a partial graph mapping from $G_{1}$ to $G_{2}$ such that $F_{2}=\left\langle F_{1 \mathbb{V}}, F_{1 \mathbb{E}}+\cdot\left(e_{1} \longmapsto e_{2}\right)\right\rangle$ and if $F_{1}$ is total, then $F_{2}$ is total and if $F_{1}$ is onto, then $F_{2}$ is onto and if $F_{1}$ is one-to-one, then $F_{2}$ is one-toone. Consider $F_{3}$ being a partial graph mapping from $G_{1}$ to $G_{2}$ such that $F_{3}=\left\langle F_{1 \mathbb{V}}, F_{1 \mathbb{E}}+\cdot\left(e_{1} \longmapsto e_{2}\right)\right\rangle$ and if $F_{1}$ is directed, then $F_{3}$ is directed and if $F_{1}$ is directed-isomorphism, then $F_{3}$ is directed-isomorphism.

(156) Suppose $e_{1} \notin$ the edges of $G_{3}$ and $e_{2} \notin$ the edges of $G_{4}$ and $v_{1} \notin$ the vertices of $G_{3}$ and $v_{2} \notin$ the vertices of $G_{4}$ and $v_{3} \in \operatorname{dom}\left(F_{0 \mathbb{V}}\right)$ and $\left(F_{0 \mathbb{V}}\right)\left(v_{3}\right)=v_{4}$. Then there exists a partial graph mapping $F$ from $G_{1}$ to $G_{2}$ such that

(i) $F=\left\langle F_{0 \mathbb{V}}+\cdot\left(v_{1} \longmapsto v_{2}\right), F_{0 \mathbb{E}}+\cdot\left(e_{1} \longmapsto e_{2}\right)\right\rangle$, and

(ii) if $F_{0}$ is weak subgraph embedding, then $F$ is weak subgraph embedding, and

(iii) if $F_{0}$ is isomorphism, then $F$ is isomorphism, and

(iv) if $F_{0}$ is directed-isomorphism, then $F$ is directed-isomorphism.

The theorem is a consequence of (155).

Let us consider graphs $G_{3}, G_{4}$, a vertex $v_{3}$ of $G_{3}$, a vertex $v_{4}$ of $G_{4}$, objects $e_{1}, e_{2}, v_{1}, v_{2}$, a supergraph $G_{1}$ of $G_{3}$ extended by $v_{3}, v_{1}$ and $e_{1}$ between them, a supergraph $G_{2}$ of $G_{4}$ extended by $v_{4}, v_{2}$ and $e_{2}$ between them, and a partial graph mapping $F_{0}$ from $G_{3}$ to $G_{4}$. Now we state the propositions:

(157) Suppose $e_{1} \notin$ the edges of $G_{3}$ and $e_{2} \notin$ the edges of $G_{4}$ and $v_{1} \notin$ the vertices of $G_{3}$ and $v_{2} \notin$ the vertices of $G_{4}$ and $v_{3} \in \operatorname{dom}\left(F_{0 \mathbb{V}}\right)$ and $\left(F_{0 \mathbb{V}}\right)\left(v_{3}\right)=v_{4}$. Then there exists a partial graph mapping $F$ from $G_{1}$ to $G_{2}$ such that

(i) $F=\left\langle F_{0 \mathbb{V}}+\cdot\left(v_{1} \longmapsto v_{2}\right), F_{0 \mathbb{E}}+\cdot\left(e_{1} \longmapsto e_{2}\right)\right\rangle$, and

(ii) if $F_{0}$ is total, then $F$ is total, and

(iii) if $F_{0}$ is onto, then $F$ is onto, and

(iv) if $F_{0}$ is one-to-one, then $F$ is one-to-one, and

(v) if $F_{0}$ is directed, then $F$ is directed.

Proof: Consider $G_{5}$ being a supergraph of $G_{3}$ extended by $v_{1}$ such that $G_{1}$ is a supergraph of $G_{5}$ extended by $e_{1}$ between vertices $v_{3}$ and $v_{1}$. 
Consider $G_{6}$ being a supergraph of $G_{4}$ extended by $v_{2}$ such that $G_{2}$ is a supergraph of $G_{6}$ extended by $e_{2}$ between vertices $v_{4}$ and $v_{2}$.

Consider $F_{1}$ being a partial graph mapping from $G_{5}$ to $G_{6}$ such that $F_{1}=\left\langle F_{0 \mathbb{V}}+\cdot\left(v_{1} \longmapsto v_{2}\right), F_{0 \mathbb{E}}\right\rangle$ and if $F_{0}$ is total, then $F_{1}$ is total and if $F_{0}$ is onto, then $F_{1}$ is onto and if $F_{0}$ is one-to-one, then $F_{1}$ is one-to-one and if $F_{0}$ is directed, then $F_{1}$ is directed and if $F_{0}$ is semi-continuous, then $F_{1}$ is semi-continuous and if $F_{0}$ is continuous, then $F_{1}$ is continuous and if $F_{0}$ is semi-directed-continuous, then $F_{1}$ is semi-directed-continuous and if $F_{0}$ is directed-continuous, then $F_{1}$ is directed-continuous. $v_{1}, v_{3} \in \operatorname{dom}\left(F_{1 \mathbb{V}}\right)$ and $\left(F_{1 \mathbb{V}}\right)\left(v_{1}\right)=v_{2}$ and $\left(F_{1 \mathbb{V}}\right)\left(v_{3}\right)=v_{4}$.

Consider $F_{2}$ being a partial graph mapping from $G_{1}$ to $G_{2}$ such that $F_{2}=\left\langle F_{1 \mathbb{V}}, F_{1 \mathbb{E}}+\cdot\left(e_{1} \longmapsto e_{2}\right)\right\rangle$ and if $F_{1}$ is total, then $F_{2}$ is total and if $F_{1}$ is onto, then $F_{2}$ is onto and if $F_{1}$ is one-to-one, then $F_{2}$ is one-toone. Consider $F_{3}$ being a partial graph mapping from $G_{1}$ to $G_{2}$ such that $F_{3}=\left\langle F_{1 \mathbb{V}}, F_{1 \mathbb{E}}+\cdot\left(e_{1} \longmapsto e_{2}\right)\right\rangle$ and if $F_{1}$ is directed, then $F_{3}$ is directed and if $F_{1}$ is directed-isomorphism, then $F_{3}$ is directed-isomorphism.

(158) Suppose $e_{1} \notin$ the edges of $G_{3}$ and $e_{2} \notin$ the edges of $G_{4}$ and $v_{1} \notin$ the vertices of $G_{3}$ and $v_{2} \notin$ the vertices of $G_{4}$ and $v_{3} \in \operatorname{dom}\left(F_{0 \mathbb{V}}\right)$ and $\left(F_{0 \mathbb{V}}\right)\left(v_{3}\right)=v_{4}$. Then there exists a partial graph mapping $F$ from $G_{1}$ to $G_{2}$ such that

(i) $F=\left\langle F_{0 \mathbb{V}}+\cdot\left(v_{1} \longmapsto v_{2}\right), F_{0 \mathbb{E}}+\cdot\left(e_{1} \longmapsto e_{2}\right)\right\rangle$, and

(ii) if $F_{0}$ is weak subgraph embedding, then $F$ is weak subgraph embedding, and

(iii) if $F_{0}$ is isomorphism, then $F$ is isomorphism, and

(iv) if $F_{0}$ is directed-isomorphism, then $F$ is directed-isomorphism.

The theorem is a consequence of (157).

(159) Let us consider graphs $G_{3}, G_{4}$, a vertex $v_{3}$ of $G_{3}$, a vertex $v_{4}$ of $G_{4}$, objects $e_{1}, e_{2}, v_{1}, v_{2}$, a supergraph $G_{1}$ of $G_{3}$ extended by $v_{1}, v_{3}$ and $e_{1}$ between them, a supergraph $G_{2}$ of $G_{4}$ extended by $v_{4}, v_{2}$ and $e_{2}$ between them, and a partial graph mapping $F_{0}$ from $G_{3}$ to $G_{4}$. Suppose $e_{1} \notin$ the edges of $G_{3}$ and $e_{2} \notin$ the edges of $G_{4}$ and $v_{1} \notin$ the vertices of $G_{3}$ and $v_{2} \notin$ the vertices of $G_{4}$ and $v_{3} \in \operatorname{dom}\left(F_{0 \mathbb{V}}\right)$ and $\left(F_{0 \mathbb{V}}\right)\left(v_{3}\right)=v_{4}$. Then there exists a partial graph mapping $F$ from $G_{1}$ to $G_{2}$ such that

(i) $F=\left\langle F_{0 \mathbb{V}}+\cdot\left(v_{1} \longmapsto v_{2}\right), F_{0 \mathbb{E}}+\cdot\left(e_{1} \longmapsto e_{2}\right)\right\rangle$, and

(ii) if $F_{0}$ is total, then $F$ is total, and

(iii) if $F_{0}$ is onto, then $F$ is onto, and

(iv) if $F_{0}$ is one-to-one, then $F$ is one-to-one, and 
(v) if $F_{0}$ is weak subgraph embedding, then $F$ is weak subgraph embedding, and

(vi) if $F_{0}$ is isomorphism, then $F$ is isomorphism.

Proof: Consider $G_{5}$ being a supergraph of $G_{3}$ extended by $v_{1}$ such that $G_{1}$ is a supergraph of $G_{5}$ extended by $e_{1}$ between vertices $v_{1}$ and $v_{3}$. Consider $G_{6}$ being a supergraph of $G_{4}$ extended by $v_{2}$ such that $G_{2}$ is a supergraph of $G_{6}$ extended by $e_{2}$ between vertices $v_{4}$ and $v_{2}$.

Consider $F_{1}$ being a partial graph mapping from $G_{5}$ to $G_{6}$ such that $F_{1}=\left\langle F_{0 \mathbb{V}}+\cdot\left(v_{1} \longmapsto v_{2}\right), F_{0 \mathbb{E}}\right\rangle$ and if $F_{0}$ is total, then $F_{1}$ is total and if $F_{0}$ is onto, then $F_{1}$ is onto and if $F_{0}$ is one-to-one, then $F_{1}$ is one-to-one and if $F_{0}$ is directed, then $F_{1}$ is directed and if $F_{0}$ is semi-continuous, then $F_{1}$ is semi-continuous and if $F_{0}$ is continuous, then $F_{1}$ is continuous and if $F_{0}$ is semi-directed-continuous, then $F_{1}$ is semi-directed-continuous and if $F_{0}$ is directed-continuous, then $F_{1}$ is directed-continuous. $v_{1}, v_{3} \in \operatorname{dom}\left(F_{1 \mathbb{V}}\right)$ and $\left(F_{1 \mathbb{V}}\right)\left(v_{1}\right)=v_{2}$ and $\left(F_{1 \mathbb{V}}\right)\left(v_{3}\right)=v_{4}$.

Consider $F_{2}$ being a partial graph mapping from $G_{1}$ to $G_{2}$ such that $F_{2}=\left\langle F_{1 \mathbb{V}}, F_{1 \mathbb{E}}+\cdot\left(e_{1} \longmapsto e_{2}\right)\right\rangle$ and if $F_{1}$ is total, then $F_{2}$ is total and if $F_{1}$ is onto, then $F_{2}$ is onto and if $F_{1}$ is one-to-one, then $F_{2}$ is one-to-one.

(160) Let us consider graphs $G_{3}, G_{4}$, a vertex $v_{3}$ of $G_{3}$, a vertex $v_{4}$ of $G_{4}$, objects $e_{1}, e_{2}, v_{1}, v_{2}$, a supergraph $G_{1}$ of $G_{3}$ extended by $v_{3}, v_{1}$ and $e_{1}$ between them, a supergraph $G_{2}$ of $G_{4}$ extended by $v_{2}, v_{4}$ and $e_{2}$ between them, and a partial graph mapping $F_{0}$ from $G_{3}$ to $G_{4}$. Suppose $e_{1} \notin$ the edges of $G_{3}$ and $e_{2} \notin$ the edges of $G_{4}$ and $v_{1} \notin$ the vertices of $G_{3}$ and $v_{2} \notin$ the vertices of $G_{4}$ and $v_{3} \in \operatorname{dom}\left(F_{0 \mathbb{V}}\right)$ and $\left(F_{0 \mathbb{V}}\right)\left(v_{3}\right)=v_{4}$. Then there exists a partial graph mapping $F$ from $G_{1}$ to $G_{2}$ such that

(i) $F=\left\langle F_{0 \mathbb{V}}+\cdot\left(v_{1} \longmapsto v_{2}\right), F_{0 \mathbb{E}}+\cdot\left(e_{1} \longmapsto e_{2}\right)\right\rangle$, and

(ii) if $F_{0}$ is total, then $F$ is total, and

(iii) if $F_{0}$ is onto, then $F$ is onto, and

(iv) if $F_{0}$ is one-to-one, then $F$ is one-to-one, and

(v) if $F_{0}$ is weak subgraph embedding, then $F$ is weak subgraph embedding, and

(vi) if $F_{0}$ is isomorphism, then $F$ is isomorphism.

Proof: Consider $G_{5}$ being a supergraph of $G_{3}$ extended by $v_{1}$ such that $G_{1}$ is a supergraph of $G_{5}$ extended by $e_{1}$ between vertices $v_{3}$ and $v_{1}$. Consider $G_{6}$ being a supergraph of $G_{4}$ extended by $v_{2}$ such that $G_{2}$ is a supergraph of $G_{6}$ extended by $e_{2}$ between vertices $v_{2}$ and $v_{4}$.

Consider $F_{1}$ being a partial graph mapping from $G_{5}$ to $G_{6}$ such that $F_{1}=\left\langle F_{0 \mathbb{V}}+\cdot\left(v_{1} \longmapsto v_{2}\right), F_{0 \mathbb{E}}\right\rangle$ and if $F_{0}$ is total, then $F_{1}$ is total and if $F_{0}$ 
is onto, then $F_{1}$ is onto and if $F_{0}$ is one-to-one, then $F_{1}$ is one-to-one and if $F_{0}$ is directed, then $F_{1}$ is directed and if $F_{0}$ is semi-continuous, then $F_{1}$ is semi-continuous and if $F_{0}$ is continuous, then $F_{1}$ is continuous and if $F_{0}$ is semi-directed-continuous, then $F_{1}$ is semi-directed-continuous and if $F_{0}$ is directed-continuous, then $F_{1}$ is directed-continuous. $v_{1}, v_{3} \in \operatorname{dom}\left(F_{1 \mathbb{V}}\right)$ and $\left(F_{1 \mathbb{V}}\right)\left(v_{1}\right)=v_{2}$ and $\left(F_{1 \mathbb{V}}\right)\left(v_{3}\right)=v_{4}$.

Consider $F_{2}$ being a partial graph mapping from $G_{1}$ to $G_{2}$ such that $F_{2}=\left\langle F_{1 \mathbb{V}}, F_{1 \mathbb{E}}+\cdot\left(e_{1} \longmapsto e_{2}\right)\right\rangle$ and if $F_{1}$ is total, then $F_{2}$ is total and if $F_{1}$ is onto, then $F_{2}$ is onto and if $F_{1}$ is one-to-one, then $F_{2}$ is one-to-one.

(161) Let us consider a graph $G$, an object $v$, a set $V$, and supergraphs $G_{1}$, $G_{2}$ of $G$ extended by vertex $v$ and edges between $v$ and $V$ of $G$. Then $G_{2}$ is $G_{1}$-isomorphic. The theorem is a consequence of (8), (53), and (143).

(162) Let us consider graphs $G_{3}, G_{4}$, objects $v_{1}, v_{2}$, sets $V_{1}, V_{2}$, a supergraph $G_{1}$ of $G_{3}$ extended by vertex $v_{1}$ and edges between $v_{1}$ and $V_{1}$ of $G_{3}$, a supergraph $G_{2}$ of $G_{4}$ extended by vertex $v_{2}$ and edges between $v_{2}$ and $V_{2}$ of $G_{4}$, and a partial graph mapping $F_{0}$ from $G_{3}$ to $G_{4}$. Suppose $V_{1} \subseteq$ the vertices of $G_{3}$ and $V_{2} \subseteq$ the vertices of $G_{4}$ and $v_{1} \notin$ the vertices of $G_{3}$ and $v_{2} \notin$ the vertices of $G_{4}$ and $F_{0 \mathbb{V}}\left\lceil V_{1}\right.$ is one-to-one and $\operatorname{dom}\left(F_{0 \mathbb{V}}\left\lceil V_{1}\right)=\right.$ $V_{1}$ and $\operatorname{rng}\left(F_{0 \mathbb{V}}\left\lceil V_{1}\right)=V_{2}\right.$. Then there exists a partial graph mapping $F$ from $G_{1}$ to $G_{2}$ such that

(i) $F_{\mathbb{V}}=F_{0 \mathbb{V}}+\cdot\left(v_{1} \longmapsto v_{2}\right)$, and

(ii) $F_{\mathbb{E}}\left\lceil\operatorname{dom}\left(F_{0 \mathbb{E}}\right)=F_{0 \mathbb{E}}\right.$, and

(iii) if $F_{0}$ is total, then $F$ is total, and

(iv) if $F_{0}$ is onto, then $F$ is onto, and

(v) if $F_{0}$ is one-to-one, then $F$ is one-to-one, and

(vi) if $F_{0}$ is weak subgraph embedding, then $F$ is weak subgraph embedding, and

(vii) if $F_{0}$ is isomorphism, then $F$ is isomorphism.

Proof: $V_{1} \subseteq \operatorname{dom}\left(F_{0 \mathbb{V}}\right)$. Set $f=F_{0 \mathbb{V}}+\cdot\left(v_{1} \longmapsto v_{2}\right)$. Consider $h_{1}$ being a function from $V_{1}$ into $G_{1}$.edgesBetween $\left(V_{1},\left\{v_{1}\right\}\right)$ such that $h_{1}$ is one-toone and onto and for every object $w$ such that $w \in V_{1}$ holds $h_{1}(w)$ joins $w$ and $v_{1}$ in $G_{1}$. Consider $h_{2}$ being a function from $V_{2}$ into $G_{2}$.edgesBetween $\left(V_{2}\right.$, $\left.\left\{v_{2}\right\}\right)$ such that $h_{2}$ is one-to-one and onto and for every object $w$ such that $w \in V_{2}$ holds $h_{2}(w)$ joins $w$ and $v_{2}$ in $G_{2}$. Set $g=F_{0 \mathbb{E}}+h_{2} \cdot\left(F_{0 \mathbb{V}}\right) \cdot\left(h_{1}{ }^{-1}\right)$. $\operatorname{dom}\left(F_{0 \mathbb{E}}\right)$ misses $\operatorname{dom}\left(h_{2} \cdot\left(F_{0 \mathbb{V}}\right) \cdot\left(h_{1}{ }^{-1}\right)\right)$. rng $F_{0 \mathbb{E}}$ misses rng $h_{2} \cdot\left(F_{0 \mathbb{V}}\right) \cdot$ $\left(h_{1}{ }^{-1}\right)$. Consider $E_{1}$ being a set such that $\overline{\overline{V_{1}}}=\overline{\overline{E_{1}}}$ and $E_{1}$ misses the edges of $G_{3}$ and the edges of $G_{1}=$ (the edges of $\left.G_{3}\right) \cup E_{1}$ and for every object $w_{1}$ such that $w_{1} \in V_{1}$ there exists an object $e_{1}$ such that $e_{1} \in E_{1}$ and $e_{1}$ 
joins $w_{1}$ and $v_{1}$ in $G_{1}$ and for every object $\tilde{e}$ such that $\tilde{e}$ joins $w_{1}$ and $v_{1}$ in $G_{1}$ holds $e_{1}=\tilde{e}$.

Consider $E_{2}$ being a set such that $\overline{\overline{V_{2}}}=\overline{\overline{E_{2}}}$ and $E_{2}$ misses the edges of $G_{4}$ and the edges of $G_{2}=$ (the edges of $\left.G_{4}\right) \cup E_{2}$ and for every object $w_{2}$ such that $w_{2} \in V_{2}$ there exists an object $e_{2}$ such that $e_{2} \in E_{2}$ and $e_{2}$ joins $w_{2}$ and $v_{2}$ in $G_{2}$ and for every object $\tilde{e}$ such that $\tilde{e}$ joins $w_{2}$ and $v_{2}$ in $G_{2}$ holds $e_{2}=\tilde{e}$. Reconsider $F=\langle f, g\rangle$ as a partial graph mapping from $G_{1}$ to $G_{2}$. If $F_{0}$ is total, then $F$ is total. If $F_{0}$ is onto, then $F$ is onto.

(163) Let us consider a graph $G_{3}$, a $G_{3}$-isomorphic graph $G_{4}$, objects $v_{1}, v_{2}$, a supergraph $G_{1}$ of $G_{3}$ extended by vertex $v_{1}$ and edges between $v_{1}$ and the vertices of $G_{3}$, and a supergraph $G_{2}$ of $G_{4}$ extended by vertex $v_{2}$ and edges between $v_{2}$ and the vertices of $G_{4}$. Suppose $v_{1} \in$ the vertices of $G_{3}$ iff $v_{2} \in$ the vertices of $G_{4}$. Then $G_{2}$ is $G_{1}$-isomorphic. The theorem is a consequence of (162) and (143).

Let us consider graphs $G_{1}, G_{2}$, a subgraph $G_{3}$ of $G_{1}$ with loops removed, a subgraph $G_{4}$ of $G_{2}$ with loops removed, and a one-to-one partial graph mapping $F_{0}$ from $G_{1}$ to $G_{2}$. Now we state the propositions:

(164) There exists a one-to-one partial graph mapping $F$ from $G_{3}$ to $G_{4}$ such that

(i) $F=F_{0}\left\lceil G_{3}\right.$, and

(ii) if $F_{0}$ is total, then $F$ is total, and

(iii) if $F_{0}$ is onto, then $F$ is onto, and

(iv) if $F_{0}$ is directed, then $F$ is directed, and

(v) if $F_{0}$ is semi-directed-continuous, then $F$ is semi-directed-continuous.

Proof: Reconsider $F=G_{4} \uparrow\left(F_{0}\left\lceil G_{3}\right)\right.$ as a one-to-one partial graph mapping from $G_{3}$ to $G_{4}$. If $F_{0}$ is total, then $F$ is total. If $F_{0}$ is onto, then $F$ is onto.

(165) There exists a one-to-one partial graph mapping $F$ from $G_{3}$ to $G_{4}$ such that

(i) $F=F_{0} \uparrow G_{3}$, and

(ii) if $F_{0}$ is weak subgraph embedding, then $F$ is weak subgraph embedding, and

(iii) if $F_{0}$ is isomorphism, then $F$ is isomorphism, and

(iv) if $F_{0}$ is directed-isomorphism, then $F$ is directed-isomorphism.

The theorem is a consequence of (164). 
(166) Let us consider a graph $G_{1}$, a $G_{1}$-isomorphic graph $G_{2}$, and a subgraph $G_{3}$ of $G_{1}$ with loops removed. Then every subgraph of $G_{2}$ with loops removed is $G_{3}$-isomorphic. The theorem is a consequence of (165).

(167) Let us consider a graph $G_{1}$, a $G_{1}$-directed-isomorphic graph $G_{2}$, and a subgraph $G_{3}$ of $G_{1}$ with loops removed. Then every subgraph of $G_{2}$ with loops removed is $G_{3}$-directed-isomorphic. The theorem is a consequence of (165).

(168) Let us consider a graph $G_{1}$, a $G_{1}$-isomorphic graph $G_{2}$, and a subgraph $G_{3}$ of $G_{1}$ with parallel edges removed. Then every subgraph of $G_{2}$ with parallel edges removed is $G_{3}$-isomorphic.

Proof: Consider $G$ being a partial graph mapping from $G_{1}$ to $G_{2}$ such that $G$ is isomorphism. Consider $E_{1}$ being a representative selection of the parallel edges of $G_{1}$ such that $G_{3}$ is a subgraph of $G_{1}$ induced by the vertices of $G_{1}$ and $E_{1}$.

Consider $E_{2}$ being a representative selection of the parallel edges of $G_{2}$ such that $G_{4}$ is a subgraph of $G_{2}$ induced by the vertices of $G_{2}$ and $E_{2}$. Define $\mathcal{P}$ [object, object] $\equiv \$_{2} \in E_{2}$ and $\left\langle \$_{1}, \$_{2}\right\rangle \in \operatorname{EdgeParEqRel}\left(G_{2}\right)$. For every objects $x, y_{1}, y_{2}$ such that $x \in$ the edges of $G_{2}$ and $\mathcal{P}\left[x, y_{1}\right]$ and $\mathcal{P}\left[x, y_{2}\right]$ holds $y_{1}=y_{2}$. For every object $x$ such that $x \in$ the edges of $G_{2}$ there exists an object $y$ such that $\mathcal{P}[x, y]$.

Consider $h$ being a function such that dom $h=$ the edges of $G_{2}$ and for every object $x$ such that $x \in$ the edges of $G_{2}$ holds $\mathcal{P}[x, h(x)]$.

(169) Let us consider a graph $G_{1}$, and subgraphs $G_{2}, G_{3}$ of $G_{1}$ with parallel edges removed. Then $G_{3}$ is $G_{2}$-isomorphic. The theorem is a consequence of (53) and (168).

(170) Let us consider a graph $G_{1}$, a $G_{1}$-directed-isomorphic graph $G_{2}$, and a subgraph $G_{3}$ of $G_{1}$ with directed-parallel edges removed. Then every subgraph of $G_{2}$ with directed-parallel edges removed is $G_{3}$-directedisomorphic.

Proof: Consider $G$ being a partial graph mapping from $G_{1}$ to $G_{2}$ such that $G$ is directed-isomorphism. Consider $E_{1}$ being a representative selection of the directed-parallel edges of $G_{1}$ such that $G_{3}$ is a subgraph of $G_{1}$ induced by the vertices of $G_{1}$ and $E_{1}$.

Consider $E_{2}$ being a representative selection of the directed-parallel edges of $G_{2}$ such that $G_{4}$ is a subgraph of $G_{2}$ induced by the vertices of $G_{2}$ and $E_{2}$. Define $\mathcal{P}$ [object, object] $\equiv \$_{2} \in E_{2}$ and $\left\langle \$_{1}, \$_{2}\right\rangle \in$ $\operatorname{DEdgeParEqRel}\left(G_{2}\right)$. For every objects $x, y_{1}, y_{2}$ such that $x \in$ the edges of $G_{2}$ and $\mathcal{P}\left[x, y_{1}\right]$ and $\mathcal{P}\left[x, y_{2}\right]$ holds $y_{1}=y_{2}$. For every object $x$ such that $x \in$ the edges of $G_{2}$ there exists an object $y$ such that $\mathcal{P}[x, y]$. 
Consider $h$ being a function such that $\operatorname{dom} h=$ the edges of $G_{2}$ and for every object $x$ such that $x \in$ the edges of $G_{2}$ holds $\mathcal{P}[x, h(x)]$.

(171) Let us consider a graph $G_{1}$, and subgraphs $G_{2}, G_{3}$ of $G_{1}$ with directedparallel edges removed. Then $G_{3}$ is $G_{2}$-directed-isomorphic. The theorem is a consequence of (53) and (170).

(172) Let us consider a graph $G_{1}$, a $G_{1}$-isomorphic graph $G_{2}$, and a simple graph $G_{3}$ of $G_{1}$. Then every simple graph of $G_{2}$ is $G_{3}$-isomorphic. The theorem is a consequence of (166) and (168).

(173) Let us consider a graph $G_{1}$, and simple graphs $G_{2}, G_{3}$ of $G_{1}$. Then $G_{3}$ is $G_{2}$-isomorphic. The theorem is a consequence of (53) and (172).

(174) Let us consider a graph $G_{1}$, a $G_{1}$-directed-isomorphic graph $G_{2}$, and a directed-simple graph $G_{3}$ of $G_{1}$. Then every directed-simple graph of $G_{2}$ is $G_{3}$-directed-isomorphic. The theorem is a consequence of (167) and (170).

(175) Let us consider a graph $G_{1}$, and directed-simple graphs $G_{2}, G_{3}$ of $G_{1}$. Then $G_{3}$ is $G_{2}$-directed-isomorphic. The theorem is a consequence of (53) and (174).

(176) Let us consider trivial, loopless graphs $G_{1}, G_{2}$, and a non empty partial graph mapping $F$ from $G_{1}$ to $G_{2}$. Then

(i) $F$ is directed-isomorphism, and

(ii) $F=\left\langle\right.$ the vertex of $G_{1} \longmapsto$ the vertex of $\left.G_{2}, \emptyset\right\rangle$.

(177) Let us consider trivial graphs $G_{1}, G_{2}$. Suppose $G_{1} \cdot \operatorname{size}()=G_{2} \cdot \operatorname{size}()$. Then there exists a partial graph mapping $F$ from $G_{1}$ to $G_{2}$ such that $F$ is directed-isomorphism. The theorem is a consequence of (31).

(178) Let us consider trivial, loopless graphs $G_{1}, G_{2}$. Then $G_{2}$ is $G_{1}$-directedisomorphic and $G_{1}$-isomorphic.

\section{REFERENCES}

[1] Grzegorz Bancerek. Introduction to trees Formalized Mathematics, 1(2):421-427, 1990.

[2] Grzegorz Bancerek, Czesław Byliński, Adam Grabowski, Artur Korniłowicz, Roman Matuszewski, Adam Naumowicz, and Karol Pąk. The role of the Mizar Mathematical Library for interactive proof development in Mizar Journal of Automated Reasoning, 61(1):9-32, 2018. do1:10.1007/s10817-017-9440-6

[3] John Adrian Bondy and U. S. R. Murty. Graph Theory. Graduate Texts in Mathematics, 244. Springer, New York, 2008. ISBN 978-1-84628-969-9.

[4] Czesław Byliński. Functions and their basic properties Formalized Mathematics, 1(1): 55-65, 1990.

[5] Czesław Byliński. Functions from a set to a set Formalized Mathematics, 1(1):153-164, 1990.

[6] Christopher David Godsil and Gordon Royle. Algebraic graph theory. Graduate Texts in Mathematics; 207. Springer, New York, 2001. ISBN 0-387-95220-9; 0-387-95241-1. 
[7] Adam Grabowski, Artur Korniłowicz, and Adam Naumowicz. Four decades of Mizar. Journal of Automated Reasoning, 55(3):191-198, 2015. doi 10.1007/s10817-015-9345-1

[8] Pavol Hell and Jaroslav Nesetril. Graphs and homomorphisms. Oxford Lecture Series in Mathematics and Its Applications; 28. Oxford University Press, Oxford, 2004. ISBN $0-19-852817-5$.

[9] Ulrich Huckenbeck. Extremal paths in graphs: foundations, search strategies, and related topics, volume 10 of Mathematical Topics. Akademie Verlag, Berlin, 1. edition, 1997. ISBN 3-05-501658-0; 978-3-05-501658-5.

[10] Tommy R. Jensen and Bjarne Toft. Graph coloring problems. Wiley-Interscience Series in Discrete Mathematics and Optimization. Wiley, New York, 1995. ISBN 0-471-02865-7.

[11] Ulrich Knauer. Algebraic graph theory: morphisms, monoids and matrices, volume 41 of De Gruyter Studies in Mathematics. Walter de Gruyter, 2011.

[12] Sebastian Koch. About supergraphs. Part I. Formalized Mathematics, 26(2):101-124, 2018. doi 10.2478/forma-2018-0009

[13] Sebastian Koch. About supergraphs. Part II. Formalized Mathematics, 26(2):125-140, 2018. doi $10.2478 /$ forma-2018-0010.

[14] Mike Krebs and Anthony Shaheen. Expander families and Cayley graphs: a beginners guide. Oxford University Press, Oxford, 2011. ISBN 0-19-976711-4; 978-0-19-976711-3.

[15] Gilbert Lee and Piotr Rudnicki. Alternative graph structures Formalized Mathematics, 13(2):235-252, 2005.

[16] Robin James Wilson. Introduction to Graph Theory. Oliver \& Boyd, Edinburgh, 1972. ISBN 0-05-002534-1.

Accepted August 29, 2019 